\title{
Conservation laws in the teleparallel theory with a positive cosmological constant
}

\author{
M. Blagojević ${ }^{1}$ and M. Vasilić ${ }^{2, *}$ \\ ${ }^{1}$ Primorska Inst. for Natural Sci. and Technology, 6000 Koper, P.O.Box 327, Slovenia \\ ${ }^{2}$ Institute of Physics, 11001 Belgrade, P.O.Box 57, Yugoslavia
}

\begin{abstract}
We study the conservation laws in the teleparallel theory with a positive cosmological constant, an extension of the teleparallel theory possessing solutions with de Sitter asymptotics. Demanding that the canonical generators of the asymptotic symmetry are well defined, we obtain their improved form, which defines the conserved charges of the theory. The physical interpretation of the results is discussed.
\end{abstract}

\section{INTRODUCTION}

The Poincaré gauge theory (PGT) is a gauge formulation of gravity which represents, at least at the classical level, a viable alternative to general relativity (GR) [1 [3]. The investigation of the canonical structure of PGT led to a significant improvement of our understanding of its gauge and physical properties [4. An important conclusion following from this analysis is that a consistent picture of the conserved charges in PGT is closely related to the asymptotic structure of spacetime [5 7], in analogy to the similar results found earlier in GR [8,9]. The conclusion is based on the study of conservation laws for the solutions which define Minkowskian geometry in the asymptotic region. We now wish to extend this type of the canonical analysis to a different class of solutions, characterized by de Sitter (dS) asymptotic geometry. Although some aspects of the related conservation laws have been already discussed in the literature [10], the canonical approach will lead us to a more clear and systematic understanding of this important problem.

We begin our investigation of the problem in a particularly interesting limit of PGT, defined by the teleparallel geometry of spacetime [11] 13]. Basic gravitational variables in PGT are the tetrad field $b_{\mu}^{i}$ and the Lorentz connection $A^{i j}{ }_{\mu}$, the related field strengths are the torsion and the curvature:

$$
T^{i}{ }_{\mu \nu}=\partial_{\mu} b^{i}{ }_{\nu}+A_{m \mu}^{i} b_{\nu}^{m}-(\mu \leftrightarrow \nu), \quad R^{i j}{ }_{\mu \nu}=\partial_{\mu} A^{i j}{ }_{\nu}+A^{i}{ }_{m \mu} A^{m j}{ }_{\nu}-(\mu \leftrightarrow \nu) .
$$

General geometric structure of PGT is described by Riemann-Cartan geometry $U_{4}$, characterized by a metric compatible connection. In the PGT context, the teleparallel (Weitzenböck) geometry $T_{4}$ is defined by the requirement of vanishing curvature:

*Email addresses: mb@phy.bg.ac.yu and mvasilic@phy.bg.ac.yu 


$$
R^{i j}{ }_{\mu \nu}(A)=0
$$

The observational relevance of the teleparallel theory (TP) is based on the existence of the one-parameter family of Lagrangians, which is empirically indistinguishable from GR. For a specific value of the parameter, the Lagrangian of the theory coincides, modulo a divergence, with the Einstein-Hilbert Lagrangian, and defines $\mathrm{GR}_{\|}$, the teleparallel form of GR [11 13].

Many important features of TP have been investigated in the literature, including its gauge structure, the canonical formulation, the initial value problem, and the form of various differential identities [14-18]. Moreover, the teleparallel approach was successfully applied in studying the tensorial proof of the positivity of energy in GR, the transparent treatment of Ashtekar's complex variables, and the construction of the teleparallel Kaluza-Klein theory [19 21. In the present paper we introduce the teleparallel theory with cosmological constant $\left(\mathrm{TP}_{\Lambda}\right)$, an extension of TP which posses solutions with the dS asymptotics. The conservation laws in $\mathrm{TP}_{\Lambda}$ are then investigated by generalizing our earlier results [22], related to the class of solutions in TP with Minkowskian asymptotics.

The value of the cosmological constant $\Lambda$ compatible with the observational data is extremely small, perhaps even zero, and leads to the fine tuning problem in the standard model of fundamental interactions [23]. The investigation of the consistency of theories with $\Lambda \neq 0$ may help us to better understand this unusual phenomenon. Thus, for instance, in GR with cosmological constant $\left(\mathrm{GR}_{\Lambda}\right)$, the perturbative stability of the ground state has been studied using a suitably defined concept of the Killing energy [24. The present paper lays the grounds for such consistency tests in $\mathrm{TP}_{\Lambda}$ [25].

The layout of the paper is as follows. In Sec. II we introduce the Lagrangian for the teleparallel theory with cosmological constant, and analyze some particular solution of the field equations. We use these results in Sec. III to choose the vacuum solution of $\mathrm{TP}_{\Lambda}$, analyze its symmetries and construct the related global symmetry generator. The vacuum solution is characterized by the tetrad field which defines the dS metric, and the non-vanishing vector component of the torsion. Its symmetry is found to be a subgroup of $S O(1,4)$ — the 3-dimensional Weyl group $W(3)$. We choose the asymptotic behaviour of all phase-space variables in Sec. IV, and use the Regge-Teitelboim method [9] to define the improved form of the global generators in Sec. V. The Hamiltonian conservation laws are presented in Sec. VI, and finally, both physical and geometric meaning of the results is discussed in Sec. VII. Some technical details are given in the Appendix.

Our conventions are the same as in Refs. [16,22].

\section{THE TELEPARALLEL THEORY WITH A COSMOLOGICAL CONSTANT}

\section{A. General structure}

In the framework of PGT, the general teleparallel theory with a cosmological constant is described by the Lagrangian density

$$
\begin{aligned}
& \mathcal{L}=b\left(\mathcal{L}_{T}-2 a \Lambda\right)+\lambda_{i j}{ }^{\mu \nu} R^{i j}{ }_{\mu \nu}+b \mathcal{L}_{M} \\
& \mathcal{L}_{T}=a\left(A T_{i j k} T^{i j k}+B T_{i j k} T^{j i k}+C T_{k} T^{k}\right) \equiv \beta_{i j k}(T) T^{i j k}
\end{aligned}
$$


Here, $\lambda_{i j}{ }^{\mu \nu}$ are Lagrange multipliers introduced to ensure the teleparallelism condition (1.1), $\Lambda$ is the cosmological constant, $a=1 / 16 \pi G$ ( $G$ is Newton's gravitational constant), $A, B$ and $C$ are free parameters, $T_{k}=T^{m}{ }_{m k}$, and $\mathcal{L}_{M}$ is the matter field Lagrangian.

By varying the Lagrangian (2.1) with respect to $b_{\mu}^{i}, A^{i j}{ }_{\mu}$ and $\lambda_{i j}{ }^{\mu \nu}$, we obtain the gravitational field equations:

$$
\begin{aligned}
& 4 \nabla_{\rho}\left(b \beta_{i}{ }^{\mu \rho}\right)-4 b \beta^{n m \mu} T_{n m i}+h_{i}{ }^{\mu} b\left(\mathcal{L}_{T}-2 a \Lambda\right)=\tau^{\mu}{ }_{i}, \\
& 4 \nabla_{\rho} \lambda_{i j}{ }^{\mu \rho}-8 b \beta_{[i j]}{ }^{\mu}=\sigma^{\mu}{ }_{i j}, \\
& R^{i j}{ }_{\mu \nu}=0
\end{aligned}
$$

where $\tau^{\mu}{ }_{i}$ and $\sigma^{\mu}{ }_{i j}$ are the energy-momentum and spin currents of matter fields, respectively.

The physical relevance of the teleparallel theory with $\Lambda=0$ is based on the fact that there is a one-parameter subclass of this theory, defined by $i) 2 A+B+C=0, C=-1$, which is empirically indistinguishable from GR [12,13]. In particular, for the parameter value ii) $B=1 / 2$ (i.e. $A=1 / 4, B=1 / 2, C=-1$ ), the one-parameter theory goes over into the so-called teleparallel formulation of GR [15], the formulation in which the gravitational field equations coincide with Einstein's equations (at least for scalar matter). After having clarified the gauge structure and conservation laws in the teleparallel theory with $\Lambda=0$ [22], we now turn our attention to the one-parameter teleparallel theory with $\Lambda \neq 0$.

\section{B. The one-parameter $\mathbf{T P}_{\Lambda}$}

We begin our study of the one-parameter $\mathrm{TP}_{\Lambda}$ by analyzing some interesting properties of its field equations, which will be useful in defining the vacuum solution of the theory. The expressions for $\beta_{i j k}$ and $\mathcal{L}_{T}$ in the one-parameter theory read:

$$
\begin{aligned}
& \beta_{i j k}=\beta_{i j k}^{\|}-\frac{a}{4}(2 B-1) \stackrel{A}{T} i j k \\
& \mathcal{L}_{T}=\mathcal{L}_{T}^{\|}-\frac{a}{12}(2 B-1) \stackrel{A}{T_{i j k}} \stackrel{A}{T}{ }^{i j k},
\end{aligned}
$$

where $\beta_{i j k}^{\|}$and $\mathcal{L}_{T}^{\|}$are the $\mathrm{GR}_{\|}$expressions, and $\stackrel{A}{T}{ }_{i j k}=T_{i j k}+T_{k i j}+T_{j k i}$. Using the identity 13

$$
a b R(A)=a b R(\Delta)+b \mathcal{L}_{T}^{\|}-\partial_{\rho}\left(2 a b T^{\rho}\right),
$$

where $R(\Delta)$ is the Riemannian scalar curvature, the Lagrangian of the one-parameter $\operatorname{TP}_{\Lambda}$ can be written in the form

$$
\mathcal{L}=-a b[R(\Delta)+2 \Lambda]-\frac{a}{12}(2 B-1) b \stackrel{A}{T}_{i j k}^{A}{ }^{A}{ }^{i j k}+\tilde{\lambda}_{i j}{ }^{\mu \nu} R^{i j}{ }_{\mu \nu}+b \mathcal{L}_{M}+\operatorname{div},
$$

with $\tilde{\lambda}_{i j}{ }^{\mu \nu} \equiv \lambda_{i j}{ }^{\mu \nu}+a H_{i j}^{\mu \nu} / 2$, and $H_{i j}^{\mu \nu}=b\left(h_{i}{ }^{\mu} h_{j}{ }^{\nu}-h_{j}{ }^{\mu} h_{i}{ }^{\nu}\right)$. The first two field equations take the form:

$$
\begin{aligned}
& R_{i k}(\Delta)-\frac{1}{2} \eta_{i k}[R(\Delta)+2 \Lambda]+\mathcal{O}(\stackrel{A}{T})=\tau_{k i} / 2 a b \\
& \nabla_{\rho}\left(\lambda_{i j}{ }^{\mu \rho}+\frac{a}{2} H_{i j}^{\mu \rho}\right)+\mathcal{O}(\stackrel{A}{T})=\sigma_{i j}^{\mu} / 4
\end{aligned}
$$


where $\mathcal{O}(\stackrel{A}{T})$ denotes terms proportional to $\stackrel{A}{T}_{i j k}$. It is now simple to conclude that for the field configurations satisfying $\stackrel{A}{T}$ ijk $=0$, the first field equation has the same form as in $G R_{\Lambda}$. The consistency of this equation requires $\tau_{k i}$ to be symmetric. Taking into account that the second field equation serves only to determine the Lagrange multipliers $\lambda_{i j}{ }^{\mu \nu}$, we can use this conclusion to generate some solutions of $\mathrm{TP}_{\Lambda}$, starting from certain solutions of $\mathrm{GR}_{\Lambda}$. Consider, for instance, a metric which has diagonal form in some coordinate system:

$$
d s^{2}=A\left(d x^{0}\right)^{2}-B_{1}\left(d x^{1}\right)^{2}-B_{2}\left(d x^{2}\right)^{2}-B_{3}\left(d x^{3}\right)^{2} .
$$

Let us choose the tetrad components to be diagonal,

$$
b_{0}^{0}=\sqrt{A}, \quad b_{\alpha}^{a}=\delta_{\alpha}^{a} \sqrt{B_{\alpha}},
$$

and fix the gauge $A_{\mu}^{i j}=0$. Then, one easily proves that $\stackrel{A}{T}_{i j k}=0$, and derives an important consequence:

If the diagonal metric (2.5a) is a solution of $\mathrm{GR}_{\Lambda}$, the related tetrad (2.5b) is a solution of the one-parameter $\mathrm{TP}_{\Lambda}$, in the gauge $A^{i j}{ }_{\mu}=0$ and with the same $\tau_{i k}$.

An important class of solutions of this type is the class of spherically symmetric solutions [12,26].

\section{Schwarzschild-de Sitter solution of $\mathbf{T P}_{\Lambda}$}

The spherically symmetric solution of $\mathrm{GR}_{\Lambda}$ in the region without matter $\left(\tau_{i k}=0\right)$ is known as the Schwarzschild-de Sitter ( $\mathrm{SdS}$ ) solution. In the isotropic coordinates $\left(t, x^{1}, x^{2}, x^{3}\right)$, it is defined by the diagonal metric

$$
A=\left(\frac{1-\psi}{1+\psi}\right)^{2}, \quad B_{1}=B_{2}=B_{3}=f^{2}(1+\psi)^{4}
$$

where

$$
\psi=m G / 2 \rho f, \quad \rho^{2}=\boldsymbol{x}^{2}, \quad f=\exp (t / \ell), \quad \ell^{2}=3 / \Lambda>0 .
$$

The gravitational field is determined by the mass parameter $m$. For $m=0$, the metric (2.6) reduces to the $\mathrm{dS}$ form (Appendix $\mathrm{A}$ ).

Let us now consider the one-parameter $\mathrm{TP}_{\Lambda}$ in the region where matter is absent $\left(\tau_{i k}, \sigma_{i k}^{\mu}=0\right)$. If we fix the gauge $A^{i j}{ }_{\mu}=0$, the preceding discussion implies that the diagonal tetrad field

$$
\tilde{b}_{\mu}^{i} \equiv\left(\begin{array}{cc}
\frac{1-\psi}{1+\psi} & 0 \\
0 & f(1+\psi)^{2} \delta_{\alpha}^{a}
\end{array}\right)
$$

is a solution of the first field equation (2.4a). In addition to that, we display here the related solution for $\lambda_{i j}{ }^{\mu \nu}$ : 


$$
\tilde{\lambda}_{i j}{ }^{\mu \nu}=-\frac{a}{2} \tilde{H}_{i j}^{\mu \nu}
$$

It is easily derived as a particular solution of the second field equation (2.4b); the general solution differs from (2.7b) by an arbitrary lambda gauge transformation.

The SdS solution (2.7) plays an important role in our study of the vacuum structure of $\mathrm{TP}_{\Lambda}$.

\section{THE VACUUM STRUCTURE OF $\mathbf{T P}_{\Lambda}$}

We begin our discussion of the conservation laws in the one-parameter $\mathrm{TP}_{\Lambda}$ by clarifying the vacuum structure of the phase space. An overview of the canonical structure of $\mathrm{TP}_{\Lambda}$, which is similar but not the same as the one of TP, is given in Appendix B.

\section{A. The dS vacuum}

For $m=0$, the $\mathrm{SdS}$ solution of $\mathrm{GR}_{\Lambda}$ reduces to the dS solution ( $\mathrm{A2}$ ),

$$
d s^{2}=d t^{2}-f^{2}\left[\left(d x^{1}\right)^{2}+\left(d x^{1}\right)^{2}+\left(d x^{1}\right)^{2}\right]=\bar{g}_{\mu \nu} d x^{\mu} d x^{\nu} .
$$

which defines, in a manner discussed above, a solution of $\mathrm{TP}_{\Lambda}$. We choose this particular solution to be the vacuum of $T P_{\Lambda}$. Thus, the vacuum values of $b^{i}{ }_{\mu}$ and $A^{i j}{ }_{\mu}$ have the form

$$
\bar{b}_{\mu}^{i}=\left(\begin{array}{cc}
1 & 0 \\
0 & f \delta_{\alpha}^{a}
\end{array}\right), \quad \bar{A}_{\mu}^{i j}=0,
$$

while the vacuum value of $\lambda_{i j}{ }^{\mu \nu}$ is given by

$$
\bar{\lambda}_{i j}{ }^{\mu \nu}=-\frac{a}{2} \bar{H}_{i j}^{\mu \nu}
$$

in accordance with (2.7).

The vacuum values of the Lagrangian variables, in conjunction with the relations defining the canonical momenta, lead to:

$$
\begin{aligned}
& \bar{\pi}_{i}{ }^{0}, \bar{\pi}_{i j}{ }^{0}, \bar{\pi}^{i j}{ }_{\mu \nu}=0, \\
& \bar{\pi}_{0}^{\gamma}=0, \quad \bar{\pi}_{a}^{\gamma}=-\frac{4 a}{\ell} f^{2} \delta_{a}^{\gamma}, \\
& \bar{\pi}_{a 0}{ }^{\alpha}=2 a f^{2} \delta_{a}^{\alpha}, \quad \bar{\pi}_{a b}{ }^{\alpha}=0 .
\end{aligned}
$$

Similarly, using the Hamiltonian field equations, we can determine the vacuum values of the Hamiltonian multipliers.

It is important to observe that our vacuum solution (3.2) is time dependent. This unusual feature will have significant influence on the canonical structure of the theory.

We now wish to clarify certain geometric aspects of the dS vacuum in the one-parameter $\mathrm{TP}_{\Lambda}$. It is clear that the relation $\bar{b}_{\mu}^{i} \bar{b}_{i \nu}=\bar{g}_{\mu \nu}$ does not fix the vacuum value of $b^{i}{ }_{\mu}$ uniquely, but only up to an arbitrary local "Lorentz" transformation $L(b)$ that acts exclusively on the 
tetrads (this transformation should be clearly distinguished from the true Lorentz transformation, which acts on all the fields in the theory). Thus, to the $\mathrm{dS}$ vacuum solution

$\bar{g}_{\mu \nu}$ of $\mathrm{GR}_{\Lambda}$, there corresponds a whole class of solutions $\bar{b}_{\mu}^{i}$ of $\mathrm{TP}_{\Lambda}$, the elements of which are related to each other by $L(b)$. Since $L(b)$ is not a gauge symmetry of the theory, all these solutions are gauge inequivalent. In certain sense, our choice (3.2) is the simplest one. To characterize this simplicity more precisely, we note that the only nonvanishing vacuum components of the torsion are

$$
\bar{T}_{0 \gamma}^{a}=\frac{1}{\ell} \delta_{\gamma}^{a} f
$$

If we define the irreducible torsion components as

$$
\begin{aligned}
& v_{i}=T_{s i}^{s}, \quad a_{i}=\frac{1}{3} \varepsilon_{i j k l} T^{j k l}, \\
& t_{i j k}=T_{(i j) k}+\frac{1}{3} \eta_{k(i} v_{j)}-\frac{1}{3} \eta_{i j} v_{k},
\end{aligned}
$$

we easily verify that their vacuum values are

$$
\bar{v}_{i}=-\frac{3}{\ell} \delta_{i}^{0}, \quad \bar{a}_{i}=\bar{t}_{i j k}=0 .
$$

Thus, our vacuum solution is characterized by the following two conditions:

$$
\begin{aligned}
& \text { a) } \bar{b}_{\mu}^{i} \bar{b}_{i \nu}=\bar{g}_{\mu \nu}, \\
& \text { b) } \bar{v}_{i}=-\frac{3}{\ell} \delta_{i}^{0}, \quad \bar{a}_{i}=\bar{t}_{i j k}=0 .
\end{aligned}
$$

The simplicity of our choice (3.2) is expressed by the geometric property that only one component of the torsion, $\bar{v}_{i}$, is different from zero.

The existence of the privileged direction $\bar{v}_{i}$ means that the vacuum (3.2) of $\mathrm{TP}_{\Lambda}$ (the teleparallel geometry) is not isotropic, in spite of the fact that the $\mathrm{dS}$ vacuum (3.1) of $\mathrm{GR}_{\Lambda}$ (Riemannian geometry) is maximally symmetric (isotropic and homogeneous). More details about the vacuum symmetries will be given in the next subsection.

In the vacuum (3.2), we have not only $\bar{a}_{i}=0$ but also $\bar{t}_{i j k}=0$. Hence, we can generalize the one-parameter Lagrangian (2.3) by adding the $t_{i j k}$ term, and still have the same vacuum solution (3.2). The resulting two-parameter theory is defined by only one condition, $2 A+$ $B+3 C=0$, which ensures the absence of the $v_{i}$ term in the action. Nevertheless, our further discussion will be restricted to the one-parameter $\operatorname{TP}_{\Lambda}$.

\section{B. Symmetries of the dS vacuum}

The action (2.1) of the general teleparallel theory is invariant under the Poincaré gauge transformations [16]. The dS vacuum (3.2) is invariant only under a subgroup of these transformations - the subgroup that leaves the vacuum configuration invariant. It is defined by demanding the following conditions on the gauge parameters:

$$
\begin{aligned}
& \omega^{i}{ }_{s} \bar{b}^{s}{ }_{\mu}-\xi^{\rho}{ }_{, \mu} \bar{b}_{\rho}^{i}-\xi^{\rho} \bar{b}_{\mu, \rho}^{i}=0, \\
& \partial_{\mu} \omega^{i j}=0, \\
& \omega_{i}{ }^{s} \bar{\lambda}_{s j}{ }^{\mu \nu}+\omega_{j}{ }^{s} \bar{\lambda}_{i s}{ }^{\mu \nu}+\xi^{\mu}{ }_{, \rho} \bar{\lambda}_{i j}{ }^{\rho \nu}+\xi^{\nu}{ }_{, \rho} \bar{\lambda}_{i j}{ }^{\mu \rho}-\partial_{\rho}\left(\xi^{\rho} \bar{\lambda}_{i j}{ }^{\mu \nu}\right)=0 .
\end{aligned}
$$


The solutions of the first two equations for $\xi^{\mu}$ and $\omega^{i j}$ are of the form

$$
\begin{array}{ll}
\xi^{0}=\varepsilon^{0}, & \xi^{\alpha}=\varepsilon^{\alpha}+\varepsilon^{\alpha}{ }_{\beta} x^{\beta}-\varepsilon^{0} \frac{1}{\ell} x^{\alpha}, \\
\omega^{0}{ }_{b}=0, & \omega^{a}{ }_{b}=\varepsilon^{a}{ }_{b},
\end{array}
$$

where $\varepsilon^{\mu}$ and $\varepsilon_{b}^{a}=-\varepsilon_{b}{ }^{a}$ are constants, and $\varepsilon^{\alpha}{ }_{\beta} \equiv \delta_{a}^{\alpha} \delta_{\beta}^{b} \varepsilon_{b}^{a}\left(\varepsilon^{\alpha \beta}=\eta^{\beta \gamma} \varepsilon_{\gamma}^{\alpha}\right)$. With these values for $\xi$ and $\omega$, the last equation in (3.4) is trivially satisfied. The global symmetry transformations of dynamical variables are obtained from their gauge transformations, derived in Ref. [16], by replacing the gauge parameters with the vacuum values (3.5). In this way we obtain

$$
\begin{aligned}
& \bar{\delta} b_{0}^{a}=\varepsilon^{a}{ }_{c} b_{0}^{c}-\varepsilon^{0} T_{0} b_{0}^{a}-\left(\varepsilon^{\gamma}+\varepsilon^{\gamma}{ }_{\beta} x^{\beta}\right) \partial_{\gamma} b_{0}^{a}, \\
& \bar{\delta} b^{a}{ }_{\alpha}=\varepsilon^{a}{ }_{c} b^{c}{ }_{\alpha}+\varepsilon_{\alpha}{ }^{\beta} b^{a}{ }_{\beta}+\varepsilon^{0}\left(\frac{1}{\ell}-T_{0}\right) b^{a}{ }_{\alpha}-\left(\varepsilon^{\gamma}+\varepsilon^{\gamma}{ }_{\beta} x^{\beta}\right) \partial_{\gamma} b^{a}{ }_{\alpha}, \\
& \bar{\delta} \pi_{0}{ }^{\alpha}=\varepsilon^{\alpha}{ }_{\beta} \pi_{0}{ }^{\beta}+\varepsilon^{0}\left(\frac{2}{\ell}-T_{0}\right) \pi_{0}{ }^{\alpha}-\left(\varepsilon^{\gamma}+\varepsilon^{\gamma}{ }_{\beta} x^{\beta}\right) \partial_{\gamma} \pi_{0}{ }^{\alpha}, \\
& \bar{\delta} \pi_{a}{ }^{\alpha}=\varepsilon_{a}{ }^{c} \pi_{c}{ }^{\alpha}+\varepsilon^{\alpha}{ }_{\beta} \pi_{a}{ }^{\beta}+\varepsilon^{0}\left(\frac{2}{\ell}-T_{0}\right) \pi_{a}{ }^{\alpha}-\left(\varepsilon^{\gamma}+\varepsilon^{\gamma}{ }_{\beta} x^{\beta}\right) \partial_{\gamma} \pi_{a}{ }^{\alpha}, \\
& \bar{\delta} \pi_{i j}{ }^{\alpha}=\varepsilon_{i}{ }^{c} \pi_{c j}{ }^{\alpha}+\varepsilon_{j}{ }^{c} \pi_{i c}{ }^{\alpha}+\varepsilon^{\alpha}{ }_{\gamma} \pi_{i j}{ }^{\gamma}+\varepsilon^{0}\left(\frac{2}{\ell}-T_{0}\right)-\left(\varepsilon^{\gamma}+\varepsilon^{\gamma}{ }_{\beta} x^{\beta}\right) \partial_{\gamma} \pi_{i j}{ }^{\alpha},
\end{aligned}
$$

where $T_{0} \equiv \partial_{0}-\left(x^{\gamma} / \ell\right) \partial_{\gamma}$, and similarly for the other variables.

The vacuum symmetry is specified by the seven parameters: $\varepsilon^{0}, \varepsilon^{\alpha}$, and $\varepsilon^{\alpha}{ }_{\beta}$. The Killing vector field $\xi=\xi^{\mu} \partial_{\mu}$ has the form

$$
\begin{aligned}
\xi & =\varepsilon^{0}\left(\partial_{0}-\frac{1}{\ell} x^{\beta} \partial_{\beta}\right)+\left(\varepsilon^{\alpha}+\varepsilon^{\alpha}{ }_{\beta} x^{\beta}\right) \partial_{\alpha} \\
& \equiv \varepsilon^{0} T_{0}+\varepsilon^{\alpha} T_{\alpha}-\frac{1}{2} \varepsilon^{\alpha \beta} L_{\alpha \beta},
\end{aligned}
$$

where $x_{\alpha}=\eta_{\alpha \beta} x^{\beta}$. The Lie algebra of the global generators reads:

$$
\begin{aligned}
& {\left[T_{0}, T_{\alpha}\right]=\frac{1}{\ell} T_{\alpha}, \quad\left[T_{\alpha}, T_{\beta}\right]=0,} \\
& {\left[L_{\alpha \beta}, T_{0}\right]=0, \quad\left[L_{\alpha \beta}, T_{\gamma}\right]=\eta_{\beta \gamma} T_{\alpha}-\eta_{\alpha \gamma} T_{\beta},} \\
& {\left[L_{\alpha \beta}, L_{\gamma \delta}\right]=\eta_{\beta \gamma} L_{\alpha \delta}-\eta_{\alpha \gamma} L_{\beta \delta}-(\gamma \leftrightarrow \delta) .}
\end{aligned}
$$

Thus, the isometry group of the $\mathrm{d} S$ vacuum is not $S O(1,4)$, as one might naively expect, but rather the subgroup that leaves the vacuum value of $v_{i}$ unchanged. According to the Lie algebra (3.7), this subgroup coincides with the three-dimensional Weyl group $W(3)$.

Since the vacuum solution depends on time, the simple time translation, generated by the vector $\varepsilon^{0} \partial_{0}$, is not the vacuum symmetry.

\section{The global symmetry generator}

The canonical generator of the local Poincaré transformations in $\mathrm{TP}_{\Lambda}$ is found in Appendix B. The global generator $G$, corresponding to the vacuum symmetry of the theory, 
is obtained by substituting the vacuum values (3.5) for $\xi$ and $\omega$ into the expression for the local Poincaré generator. Using the result (B2), we find

$$
G=-\varepsilon^{\mu} P_{\mu}+\frac{1}{2} \varepsilon^{\alpha \beta} M_{\alpha \beta},
$$

where

$$
\begin{aligned}
& P_{0}=\int d^{3} x\left(\mathcal{P}_{0}-\frac{1}{\ell} x^{\alpha} \mathcal{P}_{\alpha}\right), \quad P_{\alpha}=\int d^{3} x \mathcal{P}_{\alpha}, \\
& M_{\alpha \beta}=\int d^{3} x \mathcal{M}_{\alpha \beta}, \quad \mathcal{M}_{\alpha \beta}=x_{\alpha} \mathcal{P}_{\beta}-x_{\beta} \mathcal{P}_{\alpha}-S_{\alpha \beta},
\end{aligned}
$$

The quantities $\mathcal{P}_{\mu}$ and $S_{\alpha \beta}=S_{a b} \delta_{\alpha}^{a} \delta_{\beta}^{b}$ are defined in Appendix B. The action of the generator $G$ on a phase space functional $X$ is given by $\bar{\delta} X=\{X, G\}$.

\section{ASYMPTOTIC STRUCTURE OF SPACETIME}

In this section we discuss the behaviour of the dynamical variables at large distances, where they approach their vacuum values (3.2). We begin these considerations by identifying a set of basic (minimal) asymptotic conditions, which will be necessary for a consistent treatment of the asymptotic dynamics. Then, as a complement to these, we derive the asymptotic form of the Hamiltonian constraints. These two sets of asymptotic requirements will prove to be sufficient to determine the form of the conservation laws.

\section{A. Asymptotic conditions}

The dS space, treated as a Riemannian space, is the maximally symmetric spacetime with positive cosmological constant. Its spatial sections, determined by $t=$ const, are 3 -spheres which have no boundaries, hence there is no notion of spatial infinity. For this reason, it is not simple to define an asymptotically dS spacetime. In our approach, we rely on the analogy with the SdS form of the metric and its behaviour for $\psi \rightarrow 0$. We say that a given metric $g$ is asymptotically $\mathrm{dS}$ if

$$
g_{\mu \nu}=\bar{g}_{\mu \nu}+\mathcal{O}_{1} \text { at large } \rho \text {. }
$$

This property defines finite gravitational sources, characterized by the absence of matter at large $\rho$, where spacetime becomes nearly dS.

Thus, we are going to identify the region of large $\rho$ as the asymptotic region [27]. This is an acceptable approach if we treat our theory as an ordinary field theory, the dynamical variables of which are well defined for all values of $\rho$. It will enable us to formally apply the complete canonical apparatus to the field theory (2.3), and derive the related conservation laws. However, the geometric interpretation of this procedure will have serious limitations related to the existence of the cosmological horizon in the (vacuum) dS geometry, as discussed in Sect. VII.

Continuing with our field-theoretic approach, we now wish to determine the behaviour of the tetrad fields for large $\rho$. One could start with the general assumption $b^{i}{ }_{\mu}=\bar{b}^{i}{ }_{\mu}+\mathcal{O}_{1}$. However, for spherically symmetric solutions corresponding to finite sources, the first order 
corrections to the vacuum solution are of the SdS type. Moreover, studying the effects of the spinning matter Hayashi and Shirafuji [12] showed that the related corrections are of the second order. Thus, even in this case, the first order terms are of the SdS type. In GR, this property is always fulfilled, provided we chose convenient gauge conditions. The results of the post-Newtonian analysis of the one-parameter TP theory indicate that this might be true also for $\mathrm{TP}_{\Lambda}[12,13]$, but an independent analysis is necessary to prove this.

Motivated by these considerations, we adopt the following asymptotic conditions:

$$
b_{\mu}^{i}=\tilde{b}_{\mu}^{i}+\mathcal{O}_{2}, \quad A_{\mu}^{i j}=\hat{\mathcal{O}}
$$

where $\tilde{b}_{\mu}^{i}$ is the SdS tetrad field (2.7a), the second relation is compatible with the gauge character of $A^{i j}{ }_{\mu}$, and $\hat{\mathcal{O}}$ denotes a term with arbitrarily fast asymptotic decrease. The asymptotic behaviour of the $\lambda$ variable is assumed to have the form

$$
\lambda_{i j}{ }^{\mu \nu}=\bar{\lambda}_{i j}^{\mu \nu}+\mathcal{O}_{1}
$$

in accordance with the field equation (2.2b). In addition to this, the matter fields, being highly localized (finite gravitational sources), are chosen to have arbitrarily fast decrease.

There is a general principle which we use to define the asymptotic behaviour of dynamical variables: the expressions which vanish on shell should have an arbitrarily fast asymptotic decrease, as no solution of the field equations is thereby lost. In particular, the constraints of the theory are assumed to decrease arbitrarily fast, and consequently, all volume integrals defining the global generators (3.7) are convergent. In this way, the relations that define the canonical momenta lead to the following asymptotics:

$$
\begin{aligned}
& \pi_{i}{ }^{0}, \pi_{i j}{ }^{0}, \pi^{i j}{ }_{\mu \nu}=\hat{\mathcal{O}}, \\
& \pi_{0}{ }^{\gamma}=\mathcal{O}_{2}, \quad \pi_{a}{ }^{\gamma}=\tilde{\pi} \delta_{a}^{\gamma}+\mathcal{O}_{2}, \\
& \pi_{a 0}{ }^{\alpha}=2 a f^{2} \delta_{a}^{\alpha}+\mathcal{O}_{1}, \quad \pi_{a b}{ }^{\alpha}=\mathcal{O}_{1} .
\end{aligned}
$$

Here, and in what follows, we use the notation

$$
\begin{aligned}
& \tilde{b}^{a}{ }_{\alpha}=\tilde{v} \delta_{\alpha}^{a}, \quad \tilde{\pi}_{a}{ }^{\alpha}=\tilde{\pi} \delta_{a}^{\alpha}, \\
& \tilde{v}=f(1+\psi)^{2}, \quad \tilde{\pi}=-\frac{4 a}{\ell} \tilde{v}^{2} .
\end{aligned}
$$

In the present analysis, we shall extensively use the property of spatial derivatives to enhance asymptotic decrease: $\partial_{\alpha} \mathcal{O}_{n}=\mathcal{O}_{n+1}$. This property follows from the assumption that there are no gravitational waves in the asymptotic region, i.e. we consider isolated gravitational systems for a limited period of time.

\section{B. Asymptotic form of the constraints}

Starting with the minimal asymptotic requirements (4.1), we now turn our attention to the form of the sure constraints in the asymptotic region. The asymptotic form of $\mathcal{H}_{\alpha}, \mathcal{H}_{i j}$ and $\mathcal{H}_{\perp}$ is given by the following relations (Appendix $\mathrm{C}$ ): 


$$
\begin{aligned}
\mathcal{H}_{\alpha} & =-\partial_{\gamma} \mathcal{P}_{\alpha}^{\gamma}+\mathcal{O}_{5} \\
\mathcal{H}_{\alpha \beta} & =\mathcal{P}_{\alpha \beta}-\mathcal{P}_{\beta \alpha}+\partial_{\gamma} \pi_{\alpha \beta}{ }^{\gamma}+\mathcal{O}_{4} \\
\mathcal{H}_{0 \beta} & =f\left(\pi_{0 \beta}+\frac{4 a}{\ell} f b_{0 \beta}\right)+\partial_{\gamma} \pi_{0 \beta}{ }^{\gamma}+\mathcal{O}_{3} \\
\mathcal{H}_{\perp} & =\frac{1}{\ell} \mathcal{P}_{\alpha}{ }^{\alpha}-\partial_{\beta} \pi_{0}{ }^{\beta}+\mathcal{O}_{4}
\end{aligned}
$$

where

$$
\mathcal{P}_{\alpha}^{\gamma} \equiv b^{a}{ }_{\alpha} \pi_{a}^{\gamma}+\frac{4 a}{\ell} J \delta_{\alpha}^{\gamma}
$$

In these relations, $\mathcal{H}_{\mu \nu} \equiv \delta_{\mu}^{i} \delta_{\nu}^{j} \mathcal{H}_{i j}, \pi_{\mu \nu}{ }^{\rho} \equiv \delta_{\mu}^{i} \delta_{\nu}^{j} \pi_{i j}{ }^{\rho}, \mathcal{P}_{\alpha \beta} \equiv \eta_{\beta \gamma} \mathcal{P}_{\alpha}^{\gamma}$ and $\pi_{i \beta} \equiv \eta_{\beta \gamma} \pi_{i}^{\gamma}$. The asymptotics of $\mathcal{H}^{i j}{ }_{\alpha \beta}$ does not yield any new information, while $\phi_{i j}{ }^{\alpha}=\pi_{i j}{ }^{\alpha}-4 \lambda_{i j}{ }^{0 \alpha}$ defines the asymptotic behaviour of $\pi_{i j}{ }^{\alpha}$.

\section{IMPROVING THE GLOBAL GENERATORS}

In the Hamiltonian theory, the generators of symmetry transformations act on dynamical variables via the Poisson bracket operation, which is defined in terms of functional derivatives. A functional $F[\varphi, \pi]=\int d^{3} x f\left(\varphi, \partial_{\mu} \varphi, \pi, \partial_{\nu} \pi\right)$ has well defined functional derivatives if its variation can be written in the form

$$
\delta F=\int d^{3} x[A(x) \delta \varphi(x)+B(x) \delta \pi(x)],
$$

where terms $\delta \varphi_{, \mu}$ and $\delta \pi_{, \nu}$ are absent. In addition, the well defined phase-space functionals have to be finite on their whole domain.

The global symmetry generators (3.8) do not satisfy these requirements, although, when acting on local expressions, they produce the correct transformation laws. Our idea is to improve their form by adding appropriate surface terms, so that they can also act on global phase-space functionals. This would be a very useful achievement, since it would allow us to calculate their Poisson brackets, laying thereby the ground for our discussion of the conservation laws in the next section.

Linear momentum. We begin our discussion with the spatial translation generator $P_{\alpha}=\int d^{3} x \mathcal{P}_{\alpha}$. Its variation is given by 22

$$
\begin{aligned}
\delta \mathcal{P}_{\alpha} & =-\delta\left(b^{i}{ }_{\alpha} \pi_{i}^{\gamma}\right)_{, \gamma}+\left(\pi_{i}^{\gamma} \delta b^{i}\right)_{, \alpha}+R+\partial \hat{\mathcal{O}} \\
& =-\delta\left(b^{a}{ }_{\alpha} \pi_{a}{ }^{\gamma}\right)_{, \gamma}+\left(\pi_{a}{ }^{\gamma} \delta b^{a}{ }_{\gamma}\right)_{, \alpha}+R+\partial \mathcal{O}_{4},
\end{aligned}
$$

where $R$ denotes terms of the regular form (5.1). The second term in $\delta \mathcal{P}_{\alpha}$ can be transformed using the idea of decomposing any dynamical variable into its SdS part and the rest. This is possible owing to the adopted asymptotics (4.1). Introducing the notation

$$
\Delta b_{\gamma}^{a} \equiv b_{\gamma}^{a}-\tilde{b}_{\gamma}^{a}
$$

and similarly for the other variables, one can show that 


$$
\begin{aligned}
\pi_{a}^{\gamma} \delta b^{a}{ }_{\gamma} & =\tilde{\pi}_{a}^{\gamma} \delta \tilde{b}^{a}{ }_{\gamma}+\tilde{\pi}_{a}^{\gamma} \delta \Delta b^{a}{ }_{\gamma}+\left(\Delta \pi_{a}{ }^{\gamma}\right) \delta \tilde{b}^{a}{ }_{\gamma}+\mathcal{O}_{4} \\
& =-\frac{4 a}{\ell} \delta\left(\tilde{v}^{3}+\tilde{v}^{2} \Delta b^{a}{ }_{a}\right)+\left(\Delta \pi_{a}^{a}+\frac{8 a}{\ell} f \Delta b^{a}{ }_{a}\right) \delta \tilde{v}+\mathcal{O}_{4} \\
& =-\frac{4 a}{\ell} \delta J+\mathcal{O}_{4},
\end{aligned}
$$

where the constraint $\mathcal{H}_{\perp} \approx 0$ is used in the form (C6). Consequently,

$$
\delta \mathcal{P}_{\alpha}=-\delta \mathcal{P}_{\alpha}{ }^{\gamma}, \gamma+R+\partial \mathcal{O}_{4}
$$

and the improved linear momentum takes the form

$$
\begin{aligned}
& \tilde{P}_{\alpha}=P_{\alpha}+E_{\alpha}, \\
& E_{\alpha}=\oint d S_{\gamma} \mathcal{P}_{\alpha}{ }^{\gamma} .
\end{aligned}
$$

The constraint $\mathcal{H}_{\alpha} \approx 0$ ensures that the surface integral $E_{\alpha}$ is finite.

Angular momentum. The variation of $M_{\alpha \beta}$ has the form

$$
\begin{aligned}
& \delta M_{\alpha \beta}=\int d^{3} x \delta \mathcal{M}_{\alpha \beta}, \\
& \delta \mathcal{M}_{\alpha \beta}=x_{\alpha} \delta \mathcal{P}_{\beta}-x_{\beta} \delta \mathcal{P}_{\alpha}+\delta \mathcal{H}_{\alpha \beta}+R+\hat{\mathcal{O}}
\end{aligned}
$$

Using the expression (5.2b) for $\delta \mathcal{P}_{\alpha}$, and the relation $\delta \mathcal{H}_{\alpha \beta}=\delta \pi_{\alpha \beta}{ }^{\gamma}{ }_{\gamma}+R$, we obtain

$$
\begin{aligned}
& \tilde{M}_{\alpha \beta}=M_{\alpha \beta}+E_{\alpha \beta}, \\
& E_{\alpha \beta}=\oint d S_{\gamma}\left(x_{\alpha} \mathcal{P}_{\beta}^{\gamma}-x_{\beta} \mathcal{P}_{\alpha}^{\gamma}-\pi_{\alpha \beta}^{\gamma}\right) .
\end{aligned}
$$

The constraints $\mathcal{H}_{\alpha} \approx 0$ and $\mathcal{H}_{\alpha \beta} \approx 0$ imply that $E_{\alpha \beta}$ is finite:

$$
\begin{aligned}
& \partial_{\gamma}\left(x_{\alpha} \mathcal{P}_{\beta}^{\gamma}-x_{\beta} \mathcal{P}_{\alpha}^{\gamma}-\pi_{\alpha \beta}^{\gamma}\right) \\
& =-\left(\mathcal{P}_{\alpha \beta}-\mathcal{P}_{\beta \alpha}+\partial_{\gamma} \pi_{\alpha \beta}{ }^{\gamma}\right)+\mathcal{O}_{4}=\mathcal{O}_{4} .
\end{aligned}
$$

Killing energy. To find the improved form of $P_{0}$, the global symmetry generator which produces translations along the Killing time vector $T_{0}^{\mu}=\left(1,-x^{\alpha} / \ell\right)$, we begin with

$$
\delta P_{0}=\int d^{3} x\left(\delta \mathcal{P}_{0}-\frac{x^{\alpha}}{\ell} \delta \mathcal{P}_{\alpha}\right)
$$

The expression for $\delta \mathcal{P}_{0}$ can be found from the relations [22]

$$
\begin{aligned}
& \delta \mathcal{P}_{0}=-\delta\left(b^{k}{ }_{0} \pi_{k}{ }^{\gamma}\right)_{, \gamma}+\delta \mathcal{H}_{T}+\partial \hat{\mathcal{O}}, \\
& \delta \mathcal{H}_{T} \approx-\partial_{\alpha}\left(\frac{\partial \mathcal{L}}{\partial b^{k}{ }_{\mu, \alpha}} \delta b^{k}{ }_{\mu}\right)+\partial \hat{\mathcal{O}}+R,
\end{aligned}
$$

The first term in $\delta \mathcal{H}_{T}$ is a 3-divergence of $L^{\alpha} \equiv-4 b \beta_{i j k} h^{j \alpha} h^{k \mu} \delta b^{i}{ }_{\mu}$. Taking into account the adopted asymptotics, we find

$$
L^{\alpha}=\pi_{a}^{\alpha} \delta b^{a}{ }_{0}+\mathcal{O}_{3}=\delta\left(\pi_{a}^{\alpha} b^{a}{ }_{0}\right)+\mathcal{O}_{3}
$$


where the last equality follows from $b^{a}{ }_{0} \delta \pi_{a}{ }^{\alpha}=\mathcal{O}_{3}$. Thus,

$$
\begin{aligned}
\delta \mathcal{P}_{0} & =-\delta\left(b^{k}{ }_{0} \pi_{k}{ }^{\gamma}\right)_{, \gamma}+\delta\left(b^{a}{ }_{0} \pi_{a}{ }^{\gamma}\right)_{, \gamma}+R+\partial \mathcal{O}_{3} \\
& =-\delta\left(b^{0}{ }_{0} \pi_{0}{ }^{\gamma}\right)_{, \gamma}+R+\partial \mathcal{O}_{3} .
\end{aligned}
$$

This result, together with the relation

$$
x^{\alpha} \delta \mathcal{P}_{\alpha}=-\delta\left(x^{\alpha} \mathcal{P}_{\alpha}^{\gamma}\right)_{, \gamma}+R+\partial \mathcal{O}_{3},
$$

leads to the improved form of $P_{0}$ :

$$
\begin{aligned}
& \tilde{P}_{0}=P_{0}+E_{0} \\
& E_{0}=\oint d S_{\gamma}\left(\pi_{0}^{\gamma}-\frac{x^{\alpha}}{\ell} \mathcal{P}_{\alpha}^{\gamma}\right) .
\end{aligned}
$$

The finiteness follows from the constraints $\mathcal{H}_{\alpha} \approx 0$ and $\mathcal{H}_{\perp} \approx 0$ :

$$
\partial_{\gamma}\left(\pi_{0}^{\gamma}-\frac{x^{\alpha}}{\ell} \mathcal{P}_{\alpha}^{\gamma}\right)=\left(\partial_{\gamma} \pi_{0}^{\gamma}-\frac{1}{\ell} \mathcal{P}_{\alpha}^{\alpha}\right)+\mathcal{O}_{4}=\mathcal{O}_{4}
$$

SdS charges. As a preliminary test that our expressions for the gravitational charges $E_{\alpha}, E_{\alpha \beta}$ and $E_{0}$ are meaningful, we evaluate their values for the SdS solution. Using the constraint $\mathcal{H}_{0 \beta} \approx 0$ and the known form of the $\mathrm{SdS}$ solution, one finds

$$
\tilde{\pi}_{0}^{\alpha}=\frac{m}{4 \pi} \frac{n^{\alpha}}{\rho^{2}}+\mathcal{O}_{3} .
$$

This result, together with $\tilde{\mathcal{P}}_{\alpha}^{\gamma}=0$, leads to $\tilde{E}_{0}=m$. We also find that the SdS values of the linear and angular momentum vanish: $\tilde{E}_{\alpha}=0, \tilde{E}_{\alpha \beta}=0$.

\section{CONSERVATION LAWS}

Once we have the improved symmetry generators, it is not difficult to show their on-shell conservation, provided the time evolution is governed by the well defined Hamiltonian. The

necessary and sufficient conditions for a well defined phase-space functional $\tilde{G}[\varphi, \pi, t]$ to be a generator of global symmetries take the form [22]

$$
\frac{\partial \tilde{G}}{\partial t}+\{\tilde{G}, \tilde{H}\}=C_{P F C}, \quad\left\{\tilde{G}, \phi_{m}\right\} \approx 0,
$$

where $C_{P F C}$ is a primary first class constraint, and $\phi_{m}$ are all the constraints in the theory. The strong equality in the above relations is equality up to trivial generators; these can be, for instance, squares of constraints or pure constants (central terms). The structure of these relations has to be in agreement with the general result of Ref. [28], stating that the Poisson bracket of two well defined generators is necessarily a well defined generator. Comparing the time evolution equation for $\tilde{G}$ with the first condition in (6.1), we see that our symmetry generator is conserved only when the central terms are absent. 
In standard situations with static vacuums, the canonical temporal evolution leaves the vacuum invariant, and consequently, the Hamiltonian generates the global time translation symmetry of the vacuum. However, our present choice of the canonical time is such that we have a time dependent vacuum, which is not invariant under the action of $\tilde{H}$. As a consequence, the domain of the finite and differentiable functional $\tilde{H}$, as defined by our asymptotic conditions (4.1), is not preserved during the time evolution of the system, and therefore, strictly speaking, $\tilde{H}$ is not a well defined evolution generator. This unsatisfactory situation can be handled in either of two ways: a) we can extend our phase space by allowing a suitable class of vacuum configurations to replace our single vacuum state, or b) we could try to obtain the conservation laws working with well defined symmetry generators only. In this case, we find it much simpler to follow the second approach, which is equivalent to (6.1), but technically different. It is based on the fact that the Hamiltonian is essentially a part of the well defined global generator $\tilde{P}_{0}$, so that all the conservation laws are contained in the Poisson bracket algebra between $\tilde{P}_{0}$ and the global generators $\tilde{G}=\left(\tilde{P}_{\mu}, \tilde{M}_{\alpha \beta}\right)$.

To define this approach, we observe that our improved generators have the form of integrals of some local densities: $\tilde{P}_{0}=\int d^{3} x\left(\mathcal{G}_{0}+\mathcal{E}_{0}\right), \tilde{G}=\int d^{3} x(\mathcal{G}+\mathcal{E})$, where $\mathcal{G}$ 's are constraints. The Poisson bracket $\left\{\tilde{P}_{0}, \tilde{G}\right\}$ can be calculated in two equivalent, but technically different ways:

i) by acting with $\tilde{G}$ on the integrand of $\tilde{P}_{0}$ :

$$
\left\{\tilde{P}_{0}, \tilde{G}\right\}=\int d^{3} x\left\{\mathcal{G}_{0}+\mathcal{E}_{0}, \tilde{G}\right\} \approx \int d^{3} x\left\{\mathcal{E}_{0}, \tilde{G}\right\} \propto \int d^{3} x \bar{\delta}_{G} \mathcal{E}_{0} ;
$$

ii) by acting with $\tilde{P}_{0}$ on the integrand of $\tilde{G}$ :

$$
\left\{\tilde{P}_{0}, \tilde{G}\right\}=\int d^{3} x\left\{\tilde{P}_{0}, \mathcal{G}+\mathcal{E}\right\} \approx \int d^{3} x\left\{\tilde{P}_{0}, \mathcal{E}\right\} \propto \int d^{3} x \bar{\delta}_{1} \mathcal{E} .
$$

Here, the weak equality appears after observing that $\mathcal{G}_{0}$ and $\mathcal{G}$, being the constraints, "commute" with all symmetry generators. In the first procedure, $\bar{\delta}_{G} \mathcal{E}_{0}$ is the $\tilde{G}$ transformation of $\mathcal{E}_{0}$ which, according to the known global transformation rules (3.6), does not contain the time derivative of $\mathcal{E}_{0}$. On the other hand, the expression $\bar{\delta}_{1} \mathcal{E}$ in the second procedure is the $\tilde{P}_{0}$ transformation of $\mathcal{E}$ which does involve the time derivative of $\mathcal{E}$. The equality of the two results gives an information on the time derivative of $\tilde{G} \approx \int d^{3} x \mathcal{E}$, which will help us to find whether the generator $\tilde{G}$ is conserved or not.

To realize the two procedures just described, we need the transformation laws (3.6) and the relation

$$
\bar{\delta} \mathcal{P}_{\alpha}{ }^{\gamma}=\varepsilon_{\alpha}{ }^{\beta} \mathcal{P}_{\beta}{ }^{\gamma}+\varepsilon^{\gamma}{ }_{\beta} \mathcal{P}_{\alpha}{ }^{\beta}+\varepsilon^{0}\left(\frac{3}{\ell}-T_{0}\right)-\left(\varepsilon^{\delta}+\varepsilon^{\delta}{ }_{\beta} x^{\beta}\right) \partial_{\delta} \mathcal{P}_{\alpha}{ }^{\gamma},
$$

which is easily derived from (3.6). We also introduce the simplifying notation

$$
\bar{\delta}_{1}=\bar{\delta}\left(\varepsilon^{0}\right), \quad \bar{\delta}_{2}=\bar{\delta}\left(\varepsilon^{\alpha}\right), \quad \bar{\delta}_{3}=\bar{\delta}\left(\varepsilon^{\alpha \beta}\right),
$$

for the global transformations with parameters $\varepsilon^{0}, \varepsilon^{\alpha}$ and $\varepsilon^{\alpha \beta}$, respectively. 


\section{A. Poisson bracket algebra}

The first step in our procedure is to calculate $\left\{\tilde{P}_{0}, \tilde{G}\right\}$ using equation 6.2a). We expect to obtain relations representing the canonical realization of the part of the Weyl algebra (3.7) involving $\tilde{P}_{0}$. The first result is rather trivial:

$$
\left\{\tilde{P}_{0}, \tilde{P}_{0}\right\}=0 .
$$

Continuing with $\tilde{G}=\varepsilon^{\alpha} \tilde{P}_{\alpha}$, we find

$$
\left\{\tilde{P}_{0}, \varepsilon^{\alpha} \tilde{P}_{\alpha}\right\} \approx-\oint d S_{\gamma} \bar{\delta}_{2}\left(\pi_{0}^{\gamma}-\frac{x^{\alpha}}{\ell} \mathcal{P}_{\alpha}^{\gamma}\right) .
$$

When restricted to the $\varepsilon^{\alpha}$ parameter, the needed transformation laws are given by

$$
\bar{\delta}_{2} \pi_{0}^{\gamma}=-\varepsilon^{\beta} \partial_{\beta} \pi_{0}^{\gamma}, \quad \bar{\delta}_{2} \mathcal{P}_{\alpha}^{\gamma}=-\varepsilon^{\beta} \partial_{\beta} \mathcal{P}_{\alpha}^{\gamma} .
$$

Using the relations $\partial_{\beta} \pi_{0}^{\gamma}=\mathcal{O}_{3}$ and

$$
\begin{aligned}
-\partial_{\gamma}\left(x^{\alpha} \partial_{\beta} \mathcal{P}_{\alpha}^{\gamma}\right) & =-\partial_{\beta}\left(\mathcal{P}_{\gamma}^{\gamma}+x^{\alpha} \partial_{\gamma} \mathcal{P}_{\alpha}^{\gamma}\right)+\partial_{\gamma} \mathcal{P}_{\beta}^{\gamma} \\
& =\partial_{\gamma} \mathcal{P}_{\beta}^{\gamma}+\partial \mathcal{O}_{3},
\end{aligned}
$$

we obtain

$$
\left\{\tilde{P}_{0}, \tilde{P}_{\alpha}\right\} \approx \frac{1}{\ell} E_{\alpha} \approx \frac{1}{\ell} \tilde{P}_{\alpha}
$$

Now, we turn our attention to $\tilde{G}=\varepsilon^{\alpha \beta} \tilde{M}_{\alpha \beta} / 2$ :

$$
\left\{\tilde{P}_{0}, \frac{1}{2} \varepsilon^{\alpha \beta} \tilde{M}_{\alpha \beta}\right\} \approx \oint d S_{\gamma} \bar{\delta}_{3}\left(\pi_{0}^{\gamma}-\frac{x^{\alpha}}{\ell} \mathcal{P}_{\alpha}^{\gamma}\right),
$$

where

$$
\begin{aligned}
& \bar{\delta}_{3} \pi_{0}{ }^{\gamma}=\varepsilon^{\gamma}{ }_{\beta} \pi_{0}{ }^{\beta}-\varepsilon^{\delta}{ }_{\beta} x^{\beta} \partial_{\delta} \pi_{0}{ }^{\gamma}, \\
& \bar{\delta}_{3} \mathcal{P}_{\alpha}{ }^{\gamma}=\varepsilon_{\alpha}{ }^{\beta} \mathcal{P}_{\beta}{ }^{\gamma}+\varepsilon^{\gamma}{ }_{\beta} \mathcal{P}_{\alpha}{ }^{\beta}-\varepsilon^{\delta}{ }_{\beta} x^{\beta} \partial_{\delta} \mathcal{P}_{\alpha}{ }^{\gamma} .
\end{aligned}
$$

Using the identities

$$
\begin{aligned}
& \partial_{\gamma} \bar{\delta}_{3} \pi_{0}^{\gamma}=-\partial_{\beta}\left[\varepsilon^{\beta}{ }_{\delta} x^{\delta}\left(\partial_{\gamma} \pi_{0}^{\gamma}\right)\right], \\
& \partial_{\gamma} \bar{\delta}_{3}\left(x^{\alpha} \mathcal{P}_{\alpha}{ }^{\gamma}\right)=-\partial_{\beta}\left[\varepsilon^{\beta}{ }_{\delta} x^{\delta}\left(\mathcal{P}_{\gamma}{ }^{\gamma}+x^{\alpha} \partial_{\gamma} \mathcal{P}_{\alpha}{ }^{\gamma}\right)\right],
\end{aligned}
$$

and the constraints (4.2a) and (4.2b), we find that $\partial_{\gamma} \bar{\delta}_{3}\left(\pi_{0}^{\gamma}-x^{\alpha} \mathcal{P}_{\alpha}^{\gamma} / \ell\right)=\partial \mathcal{O}_{3}$, and consequently, the surface integral vanishes. Therefore,

$$
\left\{\tilde{M}_{\alpha \beta}, \tilde{P}_{0}\right\} \approx 0 .
$$

The results (6.3) represent the part of the Poisson bracket algebra of the global generators that involves $\tilde{P}_{0}$, and is in complete agreement with (3.7). The same kind of calculations would give us the rest of the algebra, but we don't need it here. 


\section{B. Conserved charges}

We now perform similar calculations based on the second procedure defined in (6.2b), with the conclusion that our gravitational charges are conserved.

Killing energy. For $\tilde{G}=\tilde{P}_{0}$, we have

$$
\left\{\varepsilon^{0} \tilde{P}_{0}, \tilde{P}_{0}\right\} \approx-\oint d S_{\gamma} \bar{\delta}_{1}\left(\pi_{0}^{\gamma}-\frac{x^{\alpha}}{\ell} \mathcal{P}_{\alpha}^{\gamma}\right) .
$$

The needed transformation rules are

$$
\begin{aligned}
& -\bar{\delta}_{1} \pi_{0}^{\gamma}=\varepsilon^{0} \partial_{0} \pi_{0}^{\gamma}+\frac{1}{\ell} \varepsilon^{0}\left[\pi_{0}^{\gamma}-\partial_{\beta}\left(x^{\beta} \pi_{0}^{\gamma}\right)\right], \\
& \bar{\delta}_{1}\left(x^{\alpha} \mathcal{P}_{\alpha}^{\gamma}\right)=-\varepsilon^{0} x^{\alpha} \partial_{0} \mathcal{P}_{\alpha}^{\gamma}+\frac{1}{\ell} \varepsilon^{0}\left[x^{\alpha} \partial_{\beta}\left(x^{\beta} \mathcal{P}_{\alpha}^{\gamma}\right)\right] .
\end{aligned}
$$

Then, after combining the identities

$$
\begin{aligned}
& \partial_{\gamma}\left[\pi_{0}^{\gamma}-\partial_{\beta}\left(x^{\beta} \pi_{0}^{\gamma}\right)\right]=-\partial_{\beta}\left(x^{\beta} \partial_{\gamma} \pi_{0}^{\gamma}\right), \\
& \partial_{\gamma}\left[x^{\alpha} \partial_{\beta}\left(x^{\beta} \mathcal{P}_{\alpha}^{\gamma}\right)\right]=\partial_{\beta}\left(x^{\beta} \mathcal{P}_{\gamma}^{\gamma}\right)+\partial_{\beta}\left(x^{\alpha} x^{\beta} \partial_{\gamma} \mathcal{P}_{\alpha}^{\gamma}\right)
\end{aligned}
$$

with the constraints (4.2a) and (4.2d), we obtain

$$
-\oint d S_{\gamma} \bar{\delta}_{1}\left(\pi_{0}^{\gamma}-\frac{x^{\alpha}}{\ell} \mathcal{P}_{\alpha}^{\gamma}\right)=\varepsilon^{0} \partial_{0} \oint d S_{\gamma}\left(\pi_{0}^{\gamma}-\frac{x^{\alpha}}{\ell} \mathcal{P}_{\alpha}^{\gamma}\right) .
$$

Therefore,

$$
\left\{\tilde{P}_{0}, \tilde{P}_{0}\right\} \approx \dot{E}_{0},
$$

and the relation (6.3a) implies that the Killing energy is conserved:

$$
\dot{E}_{0} \approx 0 \text {. }
$$

Linear momentum. For $\tilde{G}=\tilde{P}_{\alpha}$ in $6.2 \mathrm{~b}$, we have

$$
\begin{aligned}
& \left\{\varepsilon^{0} \tilde{P}_{0}, \tilde{P}_{\alpha}\right\} \approx \oint d S_{\gamma} \bar{\delta}_{1} \mathcal{P}_{\alpha}^{\gamma}, \\
& \bar{\delta}_{1} \mathcal{P}_{\alpha}^{\gamma}=-\varepsilon^{0} \partial_{0} \mathcal{P}_{\alpha}^{\gamma}+\frac{1}{\ell} \varepsilon^{0} \partial_{\beta}\left(x^{\beta} \mathcal{P}_{\alpha}^{\gamma}\right) .
\end{aligned}
$$

Now, the identity

$$
\partial_{\gamma}\left[\partial_{\beta}\left(x^{\beta} \mathcal{P}_{\alpha}^{\gamma}\right)\right]=\partial_{\gamma} \mathcal{P}_{\alpha}^{\gamma}+\partial_{\beta}\left(x^{\beta} \partial_{\gamma} \mathcal{P}_{\alpha}^{\gamma}\right)=\partial_{\gamma} \mathcal{P}_{\alpha}^{\gamma}+\partial \mathcal{O}_{4}
$$

leads to

$$
\left\{\tilde{P}_{0}, \tilde{P}_{\alpha}\right\} \approx-\dot{E}_{\alpha}+\frac{1}{\ell} E_{\alpha} .
$$

This relation together with $(\underline{6.3 \mathrm{~b}})$ implies the conservation of the linear momentum: 


$$
\dot{E}_{\alpha} \approx 0
$$

Angular momentum. To verify the conservation of $\tilde{M}_{\alpha \beta}$, we start with

$$
\left\{\varepsilon^{0} \tilde{P}_{0}, \tilde{M}_{\alpha \beta}\right\} \approx \oint d S_{\gamma} \bar{\delta}_{1}\left(x_{\alpha} \mathcal{P}_{\beta}^{\gamma}-x_{\beta} \mathcal{P}_{\alpha}^{\gamma}-\pi_{\alpha \beta}{ }^{\gamma}\right)
$$

where

$$
\begin{aligned}
& \bar{\delta}_{1}\left(x_{\alpha} \mathcal{P}_{\beta}^{\gamma}\right)-(\alpha \leftrightarrow \beta)=\varepsilon^{0} x_{\alpha}\left[-\partial_{0} \mathcal{P}_{\beta}^{\gamma}+\frac{1}{\ell} \partial_{\delta}\left(x^{\delta} \mathcal{P}_{\beta}^{\gamma}\right)\right]-(\alpha \leftrightarrow \beta), \\
& \bar{\delta}_{1} \pi_{\alpha \beta}{ }^{\gamma}=\varepsilon^{0}\left[-\partial_{0} \pi_{\alpha \beta} \gamma+\frac{1}{\ell}\left(2+x^{\delta} \partial_{\delta}\right) \pi_{\alpha \beta} \gamma\right]
\end{aligned}
$$

We transform these expressions using the identities

$$
\begin{aligned}
& \partial_{\gamma}\left[x_{\alpha} \partial_{\delta}\left(x^{\delta} \mathcal{P}_{\beta}^{\gamma}\right)\right]-(\alpha \leftrightarrow \beta)=-\partial_{\delta}\left[x^{\delta}\left(\mathcal{P}_{\alpha \beta}-\mathcal{P}_{\beta \alpha}\right)\right]+\partial \mathcal{O}_{3}, \\
& -\partial_{\gamma}\left[\left(2+x^{\delta} \partial_{\delta}\right) \pi_{\alpha \beta}{ }^{\gamma}\right]=-\partial_{\delta}\left(x^{\delta} \partial_{\gamma} \pi_{\alpha \beta}{ }^{\gamma}\right),
\end{aligned}
$$

where the first equality follows with the help of (4.2a). As a consequence of the constraint (4.2b), the sum of these two terms takes the trivial value $\partial \mathcal{O}_{3}$, so that

$$
\left\{\tilde{P}_{0}, \tilde{M}_{\alpha \beta}\right\} \approx-\dot{E}_{\alpha \beta} .
$$

Then, the angular momentum conservation easily follows from (6.30):

$$
\dot{E}_{\alpha \beta} \approx 0 \text {. }
$$

\section{Lagrangian formalism}

After having discussed the canonical conservation laws, we now derive the Lagrangian form of the gravitational charges. A straightforward but lengthy calculation (Appendix D) leads to the following relations:

$$
\begin{aligned}
& \mathcal{P}_{\alpha}^{\gamma}=\hat{h}_{\alpha}{ }^{0 \gamma}+\mathcal{O}_{3}, \\
& \pi_{0}^{\gamma}-\frac{x^{\alpha}}{\ell} \mathcal{P}_{\alpha}^{\gamma}=\hat{h}^{00 \gamma}-\frac{x^{\alpha}}{\ell} \hat{h}_{\alpha}{ }^{0 \gamma}+\mathcal{O}^{[\alpha \gamma]}, \alpha+\mathcal{O}_{3},
\end{aligned}
$$

where $\mathcal{O}^{[\alpha \gamma]}$ stands for an antisymmetric quantity, whose divergence gives no contribution to the surface integral. The quantity $\hat{h}^{\mu 0 \lambda}$ is defined by

$$
\begin{aligned}
\hat{h}^{\mu 0 \lambda} & \equiv \frac{1}{\sqrt{-g}} h^{\mu 0 \lambda}+\frac{4 a}{\ell} J\left(g^{\mu \lambda}-\frac{1}{2} g^{0 \mu} g^{0 \lambda}\right)-a(2 B-1) J \stackrel{A}{T} \mu 0 \lambda \\
h^{\mu \nu \lambda} & \equiv \partial_{\rho} \psi^{\mu \nu \lambda \rho}, \quad \psi^{\mu \nu \lambda \rho} \equiv a(-g)\left(g^{\mu \nu} g^{\lambda \rho}-g^{\mu \lambda} g^{\nu \rho}\right),
\end{aligned}
$$

and its indices are lowered by the true metric $g_{\mu \nu}$. Thus, all terms except $\stackrel{A}{T}^{\mu 0 \lambda}$ are expressed through the metric $\left(J^{2}=-g g^{00}+\mathcal{O}_{4}\right)$. These formulas enable us to express our canonical gravitational charges in the Lagrangian form: 


$$
\begin{aligned}
& E_{\alpha}=\oint d S_{\gamma} \hat{h}_{\alpha}{ }^{0 \gamma} \\
& E_{0}=\oint d S_{\gamma}\left(\hat{h}^{00 \gamma}-\frac{x^{\alpha}}{\ell} \hat{h}_{\alpha}{ }^{0 \gamma}\right) \\
& E_{\alpha \beta}=\oint d S_{\gamma}\left[x_{\alpha} \hat{h}_{\beta}{ }^{0 \gamma}-x_{\beta} \hat{h}_{\alpha}{ }^{0 \gamma}+\frac{f}{(-g)} \psi_{\alpha \beta 0}^{\gamma}-4\left(\lambda_{\alpha \beta}{ }^{0 \gamma}+\frac{a}{2} H_{\alpha \beta}^{0 \gamma}\right)\right] .
\end{aligned}
$$

In order to compare these results with the related calculations in the teleparallel theory with $\Lambda=0$ [22], one should consider the limit $\ell \rightarrow \infty$. Note that the asymptotic conditions are also changed in this limit. Effectively, the required transition in (6.8) is achieved by making the replacements

$$
\frac{1}{\ell} \rightarrow 0, \quad \frac{f}{(-g)} \rightarrow 1, \quad \hat{h}^{\mu 0 \lambda} \rightarrow h^{\mu 0 \lambda}-a(2 B-1) J \stackrel{A}{T}^{\mu 0 \lambda},
$$

whereupon we find a complete agreement with Ref. [22].

An alternative form of the Lagrangian formulas (6.8) can be found by introducing a different notation: $g_{\alpha \beta}=f^{2}\left(\eta_{\alpha \beta}+G_{\alpha \beta}\right), g_{00}=1+G_{00}$. Thus, for instance, the linear momentum in the limit $2 B-1=0\left(\mathrm{GR}_{\|}\right)$has the form

$$
E_{\alpha}=a f^{3} \oint d S_{\gamma}\left[\frac{2}{\ell}\left(G_{0}^{0} \delta_{\alpha}^{\gamma}-G_{\alpha}^{\gamma}\right)+f^{-2} \partial_{0}\left(f^{2} G_{\alpha}^{\gamma}\right)-\delta_{\alpha}^{\gamma} \partial_{0} G^{\beta}{ }_{\beta}\right]
$$

in agreement with the related implicitly given result in Ref. [24]. Similar formulas can be derived also for $E_{0}$ and $E_{\alpha \beta}$.

\section{CONCLUDING REMARKS}

Here, we wish to comment on some geometric aspects of our treatment of the conservation laws in the teleparallel theory with the $\mathrm{dS}$ ground state.

1. It is an attractive property of the Killing time vector that it defines the symmetry of the vacuum. However, $T_{0}$ is not timelike everywhere, but only in the region where

$$
T_{0}^{2}=1-f^{2} \rho^{2} / \ell^{2}<0, \quad \text { i.e. } \quad f^{2} \rho^{2}<\ell^{2},
$$

which is the interior of the horizon of the $\mathrm{dS}$ space (Appendix A). Therefore, the Killing time can be interpreted as the physical time only within the horizon. As a consequence, $\tilde{P}_{0}$ can be interpreted as the physical energy of the system only within the horizon. The same is true for all the other global symmetry generators.

2. When the $\mathrm{dS}$ radius $\ell$ is large, the value of the Killing energy is very close to its GR value. In spite of that, the geometric restriction to the inner part of the horizon has serious consequences. In our field-theoretic canonical approach developed so far, we worked in the region of space defined by $0 \leq \rho<\infty$. In particular, the Hamiltonian and all the symmetry generators are defined in this region. However, the true geometric distance is measured by $r=f \rho$, so that, if we restrict our discussion to the region $r \leq L$ contained in the interior of the horizon, $\rho$ must be restricted to 


$$
\rho \leq \frac{L}{f} \quad(L<\ell)
$$

This condition has an essential influence on the conservation laws. Namely, our conserved charges are given as integrals over the whole region $0 \leq \rho<\infty$, and consequently, the restriction (7.2) spoils their conservation. The bigger the dS radius $\ell$, the smaller is the error we make. Thus, the restriction (7.2) implies that the very concept of measurable energy, as well as its numerical value and the conservation law, are defined only approximately. This is true for all symmetry generators, and represents a distinctive feature of the dS space [24].

3. For finite $\ell$, our expressions for the gravitational charges are exactly conserved, but they do not have a definite physical interpretation in the whole spacetime. On the other hand, after we impose the restriction (7.2) which ensures a clear physical interpretation, the charges are conserved only approximately. In the limit $\ell \rightarrow \infty$, the gravitational charges reduce to the form found earlier in the teleparallel theory with an $M_{4}$ asymptotics. In this limit, they are both exactly conserved and have a clear physical interpretation.

4. In conclusion, the results obtained in the present paper lead to a clear understanding of the conditions under which the gravitational charges in the one-parameter teleparallel theory with the $\mathrm{dS}$ vacuum, are conserved.

\section{ACKNOWLEDGMENTS}

This work was supported by the Serbian Science foundation, Yugoslavia, and by the Slovenian Science foundation, Slovenia. One of us (M.B.) would like to thank Dj. Šijački for a discussion concerning the meaning of the Lie algebra (3.7). 


\section{APPENDIX A: ON DE SITTER GEOMETRY}

In Riemannian geometry, de Sitter (dS) space is maximally symmetric spacetime with negative curvature (positive cosmological constant) [29]. It can be simply realized as a 4-dimensional hypersphere

$$
H_{4}: \quad\left(y^{0}\right)^{2}-\left(y^{1}\right)^{2}-\left(y^{2}\right)^{2}-\left(y^{3}\right)^{2}-z^{2}=-\ell^{2}
$$

embedded in a 5-dimensional Minkowski space $M_{5}$ with metric $\eta=(+,-,-,-;-)$. It has the topology $R^{1} \times S^{3}$, the signature $(+,-,-,-)$, and negative curvature, $R=-12 / \ell^{2}$. By construction, dS space has the isometry group $S O(1,4)$, and the Killing vectors in coordinates $y^{M}=\left(y^{\mu}, z\right)$ are $K_{M N}=y_{M} \partial_{N}-y_{N} \partial_{M}$ (pseudo rotations in $M_{5}$ ).

In dS space, one can not introduce the standard concept of energy, since there is no Killing vector field which is timelike everywhere. While the rotations in $M_{5}$ are spacelike, boosts are timelike only in some regions of the $\mathrm{dS}$ space. Thus, for instance, the boost $K_{05}=\left(-z, 0,0,0, y^{0}\right)$ is timelike only for $|z|>\left|y^{0}\right|$. This is an essential property of the dS space, which is related to the existence of the (cosmological) horizon, associated to a given observer. The best we can do is to introduce the concept of energy within the horizon.

a) In the pseudo-spherical coordinates

$$
\begin{array}{ll}
y^{0}=\ell \sinh t^{\prime}, & y^{1}=\ell \cosh t^{\prime} \sin \chi \sin \theta \cos \varphi, \\
z=\ell \cosh t^{\prime} \cos \chi, & y^{2}=\ell \cosh t^{\prime} \sin \chi \sin \theta \sin \varphi \\
& y^{3}=\ell \cosh t^{\prime} \sin \chi \cos \theta
\end{array}
$$

the metric on $\mathrm{H}_{4}$ becomes

$$
d s^{2}=\ell^{2} d t^{\prime 2}-\ell^{2} \cosh ^{2} t^{\prime}\left[d \chi^{2}+\sin ^{2} \chi\left(d \theta^{2}+\sin ^{2} \theta d \varphi^{2}\right)\right] .
$$

Except for the trivial singularities at $\chi=0, \pi$ and $\theta=0, \pi$, these coordinates cover the whole $\mathrm{dS}$ space. The space can be visualized as a hyperboloid (take $\chi=\theta=\pi / 2$, for simplicity). The smallest $S^{3}$ is at $t^{\prime}=0$; in the future or past, 3-spheres expand exponentially.

b) On $H_{4}$ one can also introduce the coordinates

$$
\tau=\ell \ln \frac{y^{0}+z}{\ell}, \quad x^{\alpha}=\frac{\ell y^{\alpha}}{y^{0}+z},
$$

in which the metric takes the form

$$
d s^{2}=d \tau^{2}-f^{2}\left[\left(d x^{1}\right)^{2}+\left(d x^{1}\right)^{2}+\left(d x^{1}\right)^{2}\right], \quad f \equiv e^{\tau / \ell} .
$$

These coordinates cover half of the hypersphere $H_{4}$, since $\tau$ is defined only for $y^{0}+z>0$.

c) Let us now introduce new coordinates,

$$
\begin{array}{ll}
y^{0}=\ell \cos u \sinh t, & y^{1}=\ell \sin u \sin \theta \cos \varphi, \\
z=\ell \cos u \cosh t, & y^{2}=\ell \sin u \sin \theta \sin \varphi, \\
& y^{3}=\ell \sin u \cos \theta,
\end{array}
$$

which lead to

$$
d s^{2}=\ell^{2} \cos ^{2} u d t^{2}-\ell^{2} d u^{2}-\ell^{2} \sin ^{2} u\left(d \theta^{2}+\sin ^{2} \theta d \varphi^{2}\right) .
$$


By the coordinate transformation $r=\ell \sin u$, we obtain the line element in the static (Schwarzschild) coordinates:

$$
d s^{2}=\ell^{2}\left(1-r^{2} / \ell^{2}\right) d t^{2}-\frac{d r^{2}}{1-r^{2} / \ell^{2}}-r^{2}\left(d \theta^{2}+\sin ^{2} \theta d \varphi^{2}\right) .
$$

In these coordinates, the singularity of the metric components at $r=\ell$ is clearly visible; it can be interpreted as a cosmological singularity surrounding an observer at $r=0$.

Solving the Killing equations for the dS metric (A2) one finds the set of ten Killing vectors. In particular, the Killing vector $T_{0}=\partial_{0}-(1 / \ell) x^{\alpha} \partial_{\alpha}$ describes the translation of the time coordinate $\tau$ followed by a dilatation of the spatial coordinates $x^{\alpha}$. This vector is timelike within the horizon:

$$
\left(T_{0}\right)^{2}=1-\frac{f^{2} \rho^{2}}{\ell^{2}}>0 \Rightarrow f^{2} \rho^{2}<\ell^{2} .
$$

Note that the metric components (A2) are not singular at the horizon, while those in (A3) are singular at the horizon $r=\ell$, where $T_{0}$ becomes light-like.

\section{APPENDIX B: HAMILTONIAN AND GAUGE GENERATORS}

The Hamiltonian structure of the general TP theory with $\Lambda=0$ is described in Appendix A of Ref. [22]. Here, we discuss modifications necessary for the transition $\mathrm{TP} \rightarrow \mathrm{TP}_{\Lambda}$.

The only change in the structure of the $\mathrm{TP}_{\Lambda}$ Hamiltonian comes from the presence of the cosmological constant term $-2 a b \Lambda$ in the Lagrangian (2.1). Hence, it is simple to conclude the following:

i) the primary constraints in $\mathrm{TP}_{\Lambda}$ are the same as in $\mathrm{TP}$;

ii) the new canonical Hamiltonian is obtained by the replacement $\mathcal{H}_{c} \rightarrow \mathcal{H}_{c}+2 a b \Lambda$;

iii) all the sure (i.e. always present) secondary constraints, with the exception of $\mathcal{H}_{\perp}$, remain of the same form;

iv) there are no sure tertiary constraints (since the Poincaré and $\lambda$ gauge generators in $\mathrm{TP}_{\Lambda}$ do not posses parameters with second time derivatives, as will be clear soon);

v) all the sure determined multipliers are unchanged (the related second class constraints $\phi_{i j}{ }^{\alpha}=\pi_{i j}{ }^{\alpha}-4 \lambda_{i j}{ }^{0 \alpha}$ and $\pi^{i j}{ }_{0 \beta}$ "commute" with the correction term $2 a b \Lambda$ in $\mathcal{H}_{c}$ ).

The only change in the structure of the Hamiltonian is given by the change of the dynamical component of $\mathcal{H}_{c}$ :

$$
\mathcal{H}_{\perp} \rightarrow \mathcal{H}_{\perp}+2 a J \Lambda
$$

Consequently, the form of the Poincaré gauge generator in $\mathrm{TP}_{\Lambda}$ is obtained from the corresponding generator in TP by the replacement (B1). Thus, we have:

$$
G=G(\omega)+G(\xi)
$$


where

$$
\begin{aligned}
G(\omega)= & -\frac{1}{2} \dot{\omega}^{i j} \pi_{i j}{ }^{0}-\frac{1}{2} \omega^{i j} S_{i j}, \\
G(\xi)= & -\dot{\xi}^{0}\left(b^{k}{ }_{0} \pi_{k}{ }^{0}+\frac{1}{2} A^{i j}{ }_{0} \pi_{i j}{ }^{0}+\frac{1}{4} \lambda_{i j}{ }^{\alpha \beta} \pi^{i j}{ }_{\alpha \beta}\right)-\xi^{0} \mathcal{P}_{0} \\
& -\dot{\xi}^{\alpha}\left(b^{k}{ }_{\alpha} \pi_{k}{ }^{0}+\frac{1}{2} A^{i j}{ }_{\alpha} \pi_{i j}{ }^{0}-\frac{1}{2} \lambda_{i j}{ }^{0 \beta} \pi^{i j}{ }_{\alpha \beta}\right)-\xi^{\alpha} \mathcal{P}_{\alpha} .
\end{aligned}
$$

In the above expressions, the omitted integration over a 3-dimensional spatial hypersurface is always understood, and we use the following notation:

$$
\begin{aligned}
S_{i j}= & -\mathcal{H}_{i j}+2 b_{[i 0} \pi_{j]}{ }^{0}+2 A^{s}{ }_{[i 0} \pi_{s j]}{ }^{0}+2 \lambda_{s[i}{ }^{\alpha \beta} \pi^{s}{ }_{j] \alpha \beta}, \\
\mathcal{P}_{0} \equiv & \hat{\mathcal{H}}_{T}=\mathcal{H}_{T}-\partial_{\alpha} D^{\alpha}, \\
\mathcal{P}_{\alpha}= & \mathcal{H}_{\alpha}-\frac{1}{2} A^{i j}{ }_{\alpha} \mathcal{H}_{i j}+2 \lambda_{i j}{ }^{0 \beta} \mathcal{H}^{i j}{ }_{\alpha \beta}+\pi_{k}{ }^{0} \partial_{\alpha} b^{k}{ }_{0}+\frac{1}{2} \pi_{i j}{ }^{0} \partial_{\alpha} A^{i j}{ }_{0} \\
& -\frac{1}{4} \lambda_{i j}{ }^{\beta \gamma} \partial_{\alpha} \pi^{i j}{ }_{\beta \gamma}-\frac{1}{2} \partial_{\gamma}\left(\lambda_{i j}{ }^{\beta \gamma} \pi^{i j}{ }_{\alpha \beta}\right) .
\end{aligned}
$$

with

$$
\begin{aligned}
& \mathcal{H}_{\alpha}=\pi_{i}{ }^{\beta} T^{i}{ }_{\alpha \beta}-b^{k}{ }_{\alpha} \nabla_{\beta} \pi_{k}{ }^{\beta}+\frac{1}{2} \pi^{i j}{ }_{0 \alpha} \nabla_{\beta} \lambda_{i j}{ }^{0 \beta}, \\
& \mathcal{H}_{i j}=2 \pi_{[i}{ }^{\alpha} b_{j] \alpha}+\nabla_{\alpha} \pi_{i j}{ }^{\alpha}+2 \pi^{s}{ }_{[i 0 \alpha} \lambda_{s j]}{ }^{0 \alpha}, \\
& \mathcal{H}^{i j}{ }_{\alpha \beta}=R^{i j}{ }_{\alpha \beta}-\frac{1}{2} \nabla_{[\alpha} \pi^{i j}{ }_{0 \beta]}, \\
& D^{\alpha}=b^{k}{ }_{0} \pi_{k}{ }^{\alpha}+\frac{1}{2} A^{i j}{ }_{0} \pi_{i j}{ }^{\alpha}-\frac{1}{2} \lambda_{i j}{ }^{\alpha \beta} \pi^{i j}{ }_{\alpha \beta} .
\end{aligned}
$$

The standard Poincaré gauge transformations of fields, given in Eq. (2.3) of Ref. [16], are the symmetry transformations of $\mathrm{TP}_{\Lambda}$. Following the same procedure as described in Ref. $[\mathrm{CQG}]$, one can verify that the generator (B2a) produces the correct transformation rules of all the fields and momenta. [The derivation is based on the following two observations: a) $\mathcal{H}_{T}$ does not depend on the derivatives of momenta, and b) $\dot{Q}=\left\{Q, H_{T}\right\}$ for any dynamical variable $Q$.] Hence, the above expression for $G$ is the correct generator of Poincaré gauge transformations in the general $\mathrm{TP}_{\Lambda}$ theory.

\section{APPENDIX C: ASYMPTOTIC FORM OF THE CONSTRAINTS}

In this Appendix we calculate the asymptotic form of the constraints with an accuracy which is sufficient for studying the structure of the surface terms.

Using $N=b^{0}{ }_{0}+\mathcal{O}_{4}$ and the orthogonality relations for the tetrads, one can derive the following useful formulae:

$$
\begin{aligned}
& b=b^{0}{ }_{0} J+\mathcal{O}_{4}, \quad \Delta J=\tilde{v}^{2} \Delta b^{a}{ }_{a}+\mathcal{O}_{4}, \\
& \Delta b^{0}{ }_{0}+\left(\tilde{b}^{0}{ }_{0}\right)^{2} \Delta h_{0}{ }^{0}=\mathcal{O}_{4}, \quad \Delta b^{a}{ }_{a}+\tilde{v}^{2} \Delta h_{a}{ }^{a}=\mathcal{O}_{4},
\end{aligned}
$$

where we used the notation $\Delta X \equiv X-\tilde{X}$. One should also note that the SdS solution automatically satisfies all the constraints.

1. We begin with the constraint $\mathcal{H}_{\alpha}$, written in the form

$$
\begin{aligned}
\mathcal{H}_{\alpha} & =\pi_{a}{ }^{\beta} T^{a}{ }_{\alpha \beta}-b^{a}{ }_{\alpha} \partial_{\beta} \pi_{a}{ }^{\beta}+\mathcal{O}_{5} \\
& =\pi_{a}{ }^{\beta} \partial_{\alpha} b^{a}{ }_{\beta}-\partial_{\beta}\left(b^{a}{ }_{\alpha} \pi_{a}{ }^{\beta}\right)+\mathcal{O}_{5} .
\end{aligned}
$$


Then, using the formula

$$
\Delta\left(\pi_{a}{ }^{\beta} \partial_{\alpha} b^{a}{ }_{\beta}\right)=-\frac{4 a}{\ell} \Delta\left(\partial_{\alpha} J\right)+\mathcal{O}_{5},
$$

one finds

$$
\mathcal{H}_{\alpha}=-\Delta\left(\partial_{\gamma} \mathcal{P}_{\alpha}^{\gamma}\right)+\mathcal{O}_{5}
$$

After noting that $\tilde{\mathcal{P}}_{\alpha}^{\gamma}=0$, we finally obtain

$$
\mathcal{H}_{\alpha}=-\partial_{\gamma} \mathcal{P}_{\alpha}^{\gamma}+\mathcal{O}_{5}
$$

2. Going now to $\mathcal{H}_{a c}$ and using the same technique, we find

$$
\begin{aligned}
\mathcal{H}_{a c} & =2\left(\tilde{\pi}_{[a}{ }^{\alpha} \Delta b_{c] \alpha}+\Delta \pi_{[a}{ }^{\alpha} \tilde{b}_{c] \alpha}\right)+\partial_{\alpha} \pi_{a c}{ }^{\alpha}+\mathcal{O}_{4} \\
& =2\left(\tilde{\pi} \Delta b_{[c a]}+\tilde{v} \Delta \pi_{[a c]}\right)+\partial_{\alpha} \pi_{a c}{ }^{\alpha}+\mathcal{O}_{4} .
\end{aligned}
$$

In particular,

$$
\pi_{[a c]}+\frac{4 a}{\ell} f b_{[a c]}=\mathcal{O}_{3}
$$

If we further note that

$$
\begin{aligned}
\mathcal{P}_{[a c]} & =\tilde{\pi}_{e[c} \Delta b_{a]}^{e}+\Delta \pi_{e[c} \tilde{b}_{a]}^{e}+\mathcal{O}_{4} \\
& =\tilde{\pi} \Delta b_{[c a]}+\tilde{v} \Delta \pi_{[a c]}+\mathcal{O}_{4},
\end{aligned}
$$

we verify the final expression (4.2b).

3. The constraint $\mathcal{H}_{0 c}$, after discarding $\mathcal{O}_{3}$ terms, reads

$$
\mathcal{H}_{0 c}=\pi_{0}{ }^{\alpha} \bar{b}_{c \alpha}-\bar{\pi}_{c}{ }^{\alpha} b_{0 \alpha}+\partial_{\gamma} \pi_{0 c}{ }^{\gamma}+\mathcal{O}_{3},
$$

which leads to the result (4.20).

Using the fact that $\pi_{0 c}^{\gamma}=4 \lambda_{0 c}{ }^{0 \gamma}+\hat{\mathcal{O}}=-2 a \tilde{H}_{0 c}^{0 \gamma}+\mathcal{O}_{2}$, we find

$$
\pi_{0 \beta}+\frac{4 a}{\ell} f b_{0 \beta}=8 a f \psi_{, \beta}+\mathcal{O}_{3} .
$$

Since the divergence of $\partial_{\gamma} \psi \sim n^{\gamma} / \rho^{2}$ vanishes, the preceding relation implies

$$
\partial^{\beta}\left(\pi_{0 \beta}+\frac{4 a}{\ell} f b_{0 \beta}\right)=\mathcal{O}_{4}
$$

4. Using the general definition, eq. (A1.4b) in Ref. [5], the dynamical component of the Hamiltonian takes the form

$$
N \mathcal{H}_{\perp}=\frac{1}{2} \pi_{a}{ }^{\alpha} T^{a}{ }_{0 \alpha}+2 a \Lambda b-\partial_{\beta} \pi_{0}{ }^{\beta}+\mathcal{O}_{4}
$$

The substitution $X=\tilde{X}+\Delta X$ yields

$$
N \mathcal{H}_{\perp}=\frac{f}{2 \ell} \Delta \pi_{a}^{a}-\frac{2 a}{\ell} \tilde{v}^{2}\left(\partial_{0} \Delta b^{c}{ }_{c}-\partial_{c} \Delta b_{0}^{c}\right)+2 a \Lambda \Delta b-\partial_{\beta} \pi_{0}{ }^{\beta}+\mathcal{O}_{4} .
$$


In order to eliminate the velocity $\partial_{0} \Delta b^{c}{ }_{c}$ we use the relation

$$
b_{0}^{0}{ }_{0} \pi_{a}{ }^{\alpha} b^{a}{ }_{\alpha}=-4 a J h_{c}{ }^{\gamma} T^{c}{ }_{0 \gamma}+\mathcal{O}_{4},
$$

which follows from the definition of $\pi_{a}{ }^{\alpha}$. It leads to

$$
\frac{f}{\ell}\left(\frac{1}{2} \Delta \pi_{a}{ }^{a}+\frac{8 a}{\ell} \tilde{v} \Delta b^{c}{ }_{c}\right)=2 a \Lambda \Delta b-\frac{2 a}{\ell} \tilde{v}^{2}\left(\partial_{0} \Delta b^{c}{ }_{c}-\partial_{c} \Delta b^{c}{ }_{0}\right)+\mathcal{O}_{4} .
$$

Substituting $\partial_{0} \Delta b^{c}{ }_{c}$ from this equation into $N \mathcal{H}_{\perp}$ leads to

$$
N \mathcal{H}_{\perp}=\frac{f}{\ell}\left(\Delta \pi_{a}{ }^{a}+\frac{8 a}{\ell} \tilde{v} \Delta b^{c}{ }_{c}\right)-\partial_{\beta} \pi_{0}{ }^{\beta}+\mathcal{O}_{4}
$$

Since $\partial_{\beta} \pi_{0}{ }^{\beta}=\mathcal{O}_{3}$, we have

$$
\Delta \pi_{a}^{a}+\frac{8 a}{\ell} f \Delta b_{c}^{c}=\mathcal{O}_{3}
$$

The constraint $N \mathcal{H}_{\perp}$ can be rewritten in another useful form:

$$
N \mathcal{H}_{\perp}=\frac{1}{\ell} \Delta \mathcal{P}_{\alpha}{ }^{\alpha}-\partial_{\beta} \pi_{0}{ }^{\beta}+\mathcal{O}_{4}
$$

Now, taking into account that $\tilde{\mathcal{P}}_{\alpha}{ }^{\alpha}=0$ and $N=1+\mathcal{O}_{1}$, we find

$$
\mathcal{H}_{\perp}=\frac{1}{\ell} \mathcal{P}_{\alpha}{ }^{\alpha}-\partial_{\beta} \pi_{0}{ }^{\beta}+\mathcal{O}_{4}
$$

As a consequence, $\mathcal{P}_{\alpha}{ }^{\alpha}=\mathcal{O}_{3}$.

\section{APPENDIX D: LAGRANGIAN FORM OF THE CONSERVED CHARGES}

In this Appendix, we derive equations (6.8) representing the Lagrangian form of the conserved charges. Most of the needed formulas are taken from Appendix D of Ref. [22].

Using the defining relation for the momentum variables $\pi_{i}{ }^{\mu}\left(\pi_{i}{ }^{\mu}=-4 b h^{j \mu} \beta_{i j}{ }^{0}\right)$, we straightforwardly express $\pi_{0}^{\gamma}$ and $\mathcal{P}_{\alpha}^{\gamma}$ in terms of Lagrangian variables:

$$
\begin{aligned}
\pi_{0}^{\gamma} & =2 a h^{a \gamma}\left(\partial_{\beta} H_{a 0}^{\beta 0}+\frac{2}{\ell} b h_{a}^{0}\right)+\mathcal{O}_{3} \\
\mathcal{P}_{\alpha}^{\gamma}= & -\frac{a}{\sqrt{-g}} g_{\alpha \beta}\left(\dot{H}_{c 0}^{\beta 0} H^{c 0 \gamma 0}+\dot{H}_{c 0}^{\gamma 0} H^{c 0 \beta 0}\right)+\frac{4 a}{\ell} J \delta_{\alpha}^{\gamma} \\
& +a \eta_{\alpha \beta}\left(H^{\beta \gamma 0 \delta}-f H^{\beta 0 \gamma \delta}-f H^{\gamma 0 \beta \delta}\right)_{, \delta} \\
& +a(2 B-1) g_{\alpha \beta} J \stackrel{A}{T} \beta \gamma 0+\mathcal{O}_{4} .
\end{aligned}
$$

In the above formulas, $H_{i j}^{\mu \nu}=H_{i j}{ }^{\mu \nu}, H^{\mu \nu \lambda \rho}=\delta_{i}^{\mu} \delta_{j}^{\nu} H^{i j \lambda \rho}$, and $\stackrel{A}{T}{ }^{\mu \nu \lambda}=h^{i \mu} h^{j \nu} h^{k \lambda} \stackrel{A}{T}{ }_{i j k}$. We want to compare these with the GR expressions. Rewriting the well known superpotential of Landau and Lifshitz (6.7b) in terms of tetrad fields, we obtain 


$$
\begin{gathered}
h^{00 \gamma}=2 a b h^{a \gamma} \partial_{\beta} H_{0 a}^{0 \beta}+\mathcal{O}^{[\alpha \gamma]}{ }_{, \alpha}+\mathcal{O}_{3} \\
h^{\alpha 0 \gamma}=-a\left(\dot{H}_{c 0}^{\alpha 0} H^{c 0 \gamma 0}+\dot{H}_{c 0}^{\gamma 0} H^{c 0 \alpha 0}\right)+a f\left(H^{\alpha \delta 0 \gamma}-f H^{\gamma 0 \alpha \delta}\right)_{, \delta}+\mathcal{O}_{4} .
\end{gathered}
$$

Now, comparing (D1) and (D2), and using the quantity $\hat{h}^{\mu 0 \lambda}$ defined in (6.7b) to shorten writing, we arrive at

$$
\begin{gathered}
\pi_{0}^{\gamma}=\hat{h}^{00 \gamma}+\frac{2 a}{\ell} f^{2}\left(2 h^{\gamma 0}-f g^{0 \gamma}\right)+\mathcal{O}^{[\alpha \gamma]}{ }_{\alpha}+\mathcal{O}_{3}, \\
\mathcal{P}_{\alpha}^{\gamma}=\hat{h}_{\alpha}^{0 \gamma}+a \eta_{\alpha \beta}\left(H^{\beta \gamma 0 \delta}-H^{\beta \delta 0 \gamma}-f H^{\beta 0 \gamma \delta}\right)_{, \delta}+\mathcal{O}_{4},
\end{gathered}
$$

where $\hat{h}_{\mu}{ }^{0 \lambda}=g_{\mu \nu} \hat{h}^{\nu 0 \lambda}$, and $h^{\mu \nu} \equiv \delta_{i}^{\mu} h^{i \nu}$. The second term in (D3b) is $\mathcal{O}_{3}$, and we straightforwardly verify the Lagrangian expression for the conserved momentum. To obtain the same for the Killing energy, we first notice that

$$
\eta_{\alpha \beta}\left(H^{\beta \gamma 0 \delta}-H^{\beta \delta 0 \gamma}-f H^{\beta 0 \gamma \delta}\right)_{, \delta}=f^{2}\left[\left(2 h^{\beta 0}-f g^{0 \beta}\right) \delta_{\alpha}^{\gamma}-(\beta \leftrightarrow \gamma)\right]_{, \beta}+\mathcal{O}_{4} .
$$

Using this in $\pi_{0}^{\gamma}-x^{\alpha} \mathcal{P}_{\alpha}^{\gamma} / \ell$, we verify the second formula of (6.7a), and consequently, the Lagrangian expression for the conserved Killing energy.

When applied to the angular momentum, the same procedure gives

$$
x_{\alpha} \mathcal{P}_{\beta}^{\gamma}-x_{\beta} \mathcal{P}_{\alpha}^{\gamma}=x_{\alpha} \hat{h}_{\beta}^{0 \gamma}-x_{\beta} \hat{h}_{\alpha}^{0 \gamma}+\frac{f}{(-g)} \psi_{\alpha \beta 0}^{\gamma}-2 a H_{\alpha \beta}^{0 \gamma}+\mathcal{O}_{\alpha \beta, \delta}^{[\delta \gamma]}+\mathcal{O}_{3},
$$

which leads to the Lagrangian expression for the angular momentum, as given in (6.8). 


\section{REFERENCES}

[1] T. W. B. Kibble, J. Math. Phys. 2, 212 (1961);

[2] F. W. Hehl, P. von der Heyde. D. Kerlick and J. M. Nester, Rev. Mod. Phys. 48, 393 (1976); F. W. Hehl, in Cosmology and Gravitation: Spin, Torsion, Rotation and Supergravity, eds. P. G. Bergman and V. de Sabbata (Plenum, New York, 1980) p. 5.

[3] K. Hayashi and T. Shirafuji, Prog. Theor. Phys. 64, 866 (1980); 64, 883 (1980); 64, 1435 (1980); 64, 2222 (1980).

[4] W. Szczyrba, Phys. Rev. D25, 2548 (1982); M. Blagojević and I. Nikolić, Phys. Rev. D28, 2455 (1983); I. Nikolić, Phys. Rev. D30, 2508 (1984); T. Kawai, Prog. Theor. Phys. 76, 1166 (1986); P. Baekler and E. Mielke, Fortschr. Phys. 36, 549 (1988);

[5] M. Blagojević and M. Vasilić, Class. Quant. Grav. 5, 1241 (1988).

[6] M. Blagojević, Gravitation and gauge symmetries (Institute of Physics Publishing, Bristol, 2001).

[7] K. Hayashi and T. Shirafuji, Prog. Theor. Phys. 73, 54 (1985). J. M. Nester, Some Progress in Classical Canonical Gravity, in Directions in general relativity, Proc. of the 1993 International symposium, Maryland, vol. I, papers in honour of Charles Misner, edited by B. L. Hu, M. P. Ryan and C. V. Vishveshwara (Cambridge University Press, Cambridge 1993) p. 245 (this paper gives a review of the covariant Hamiltonian approach based on differential form methods).

[8] R. Arnowitt, S. Deser and C. W. Misner, in: Gravitation - An Introduction to Current Research, ed. L. Witten, (Willey, New York, 1962) p. 227; B. DeWitt, Phys. Rev 160, 1113 (1967).

[9] T. Regge and C. Teitelboim, Ann. Phys. (N.Y) 88, 286 (1974); R. Beig and O’Murchadha, Ann. Phys. (NY) 174463 (1987);

[10] See, for instance, P. Baekler, R. Hecht, F. W. Hehl and T. Shirafuji, Prog. Theor. Phys. 78, 16 (1987); E. W. Mielke and R. P. Wallner, Nuovo Cim. B 101, 607 (1988); R. D. Hecht and J. M. Nester, Phys. Lett. A 180, 324 (1993); R. D. Hecht, Gen. Rel. Grav. 27,537 (1995); E. Mielke, Geometrodynamics of gauge fields - On the Geometry of Yang-Mills Fields and Gravitational Gauge Theories (Berlin, Akademie-Verlag, 1987).

[11] C. Møller, Ann. Phys. 12 (1961) 118; Mat. Fys. Scr. Dan. Vid. Selsk. 1, No 10 (1961); 89, No 13 (1978).

[12] K. Hayashi and T. Shirafuji, Phys. Rev. D19, 3524 (1979).

[13] J. Nitsch, in Cosmology and Gravitation: Spin, Torsion, Rotation and Supergravity, eds. P. G. Bergman and V. de Sabbata (Plenum, New York, 1980) p. 63; F. W. Hehl, J. Nietsch and P. von der Heyde, in General Relativity and Gravitation - One Hundred Years after the birth of Albert Einstein, ed. A. Held (Plenum, New York, 1980) vol. 1, p. 329.

[14] W. Kopczyński, J. Phys. A15, 493 (1982). J. M. Nester, Class. Quant. Grav. 5, 1003 (1988).

[15] W. H. Cheng, D. C. Chern and J. M. Nester, Phys. Rev. D 38, 2656 (1988); J. W. Maluf, J. Math. Phys. 36 (8), 4242 (1995). M. Blagojević and I. A. Nikolić, Phys. Rev. D62 (2000) 024021 (hep-th/0002022); M. Blagojević, Ann. Phys. (Leipzig) 10 [5], 1 (2001) (hep-th/0002022).

[16] M. Blagojević and M. Vasilić, Class. Quant. Grav. 17, 3785 (2000) (hep-th/0006080). 
[17] R. D. Hecht, J. Lemke and R. P. Wallner Phys. Rev. D44, 2442 (1991). see also R. D. Hecht, J. M. Nester and V. V. Zhytnikov Phys. Lett. A222, 37 (1996).

[18] Y. Itin, Conserved currents for general teleparallel models, e-print gr-qc/0103017; Class. Quant. Grav. 19, 173 (2002).

[19] J. M. Nester, Int. J. Mod. Phys. A4, 1755 (1989); Phys. Lett. A139, 112 (1989).

[20] E. W. Mielke, Ann. Phys. (N.Y.) 219, 78 (1992).

[21] V. C. de Andrade, L. C. T. Guillen and J. G. Pereira, Phys. Rev. D61 (2000) 084031.

[22] M. Blagojević and M. Vasilić, Phys. Rev. D64 (2001) 044010 (hep-th/0008159).

[23] E. W. Kolb and M. S. Turner, The Early Universe (Addison-Wesley, Reading, Mass., 1994)

[24] L. F. Abbot and S. Deser, Nucl. Phys. B195 (1982) 76; see also L. F. Abott and S. Deser, Phys. Lett B116 (1982) 259.

[25] For interesting consistency tests of the canonical form of a theory, see H. Chen, J. M. Nester and H. J. Yo, Acta. Phys. Pol. B29, 961 (1998).

[26] J. Nitsch and F. Hehl, Phys. Lett. 90B (1980) 98;

[27] An assymptotically dS geometry can be defined by demanding the metric to behave as the dS metric for $t \rightarrow-\infty$; see, e.g. M. Spradlin, A. Strominger and A. Volovich, Les Houches Lectures on de Sitter Space, e-print hep-th/0110007. For finite $r=\rho \exp (t / \ell)$, this is equivalent to $\rho \rightarrow \infty$.

[28] J. D. Brown and M. Henneaux, J. Math. Phys. 27 (2), 489 (1986).

[29] S. W. Hawking and G. F. R. Ellis, The Large Scale Structure of Spacetime (Cambridge Univ. Press, Cambridge, 1973). 